CRYSTALLOGRAPHIC COMMUNICATIONS

ISSN 2056-9890

Received 12 December 2016

Accepted 28 December 2016

Edited by G. Smith, Queensland University of Technology, Australia

Keywords: crystal structure; hydrogen bonding; Schiff base; $\mathrm{C}-\mathrm{H} \cdots \pi$ interactions; 9-anthraldehyde; N-phenyl-p-phenylenediamine; AMPD; anthracene.

CCDC reference: 1524849

Supporting information: this article has supporting information at journals.iucr.org/e

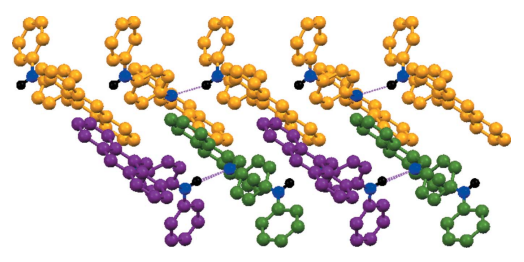

OPEN Ә ACCESS

\section{Crystal structure of $(E)-N^{1}-[($ anthracen-9-yl)- methylidene]- $N^{4}$-phenylbenzene-1,4-diamine}

\author{
Md. Serajul Haque Faizi, ${ }^{a}$ Ashanul Haque, ${ }^{a}$ Musheer Ahmad ${ }^{b}$ and Irina A. \\ Golenya $^{\text {c* }}$
}

\begin{abstract}
${ }^{a}$ Department of Chemistry, College of Science, Sultan Qaboos University, PO Box 36 Al-Khod 123, Muscat, Sultanate of Oman, 'b Department of Applied Chemistry, Aligarh Muslim University 202002 UP, India, and ${ }^{\mathbf{c}}$ National Taras Shevchenko University, Department of Chemistry, Volodymyrska str. 64, 01601 Kyiv, Ukraine. *Correspondence e-mail: igolenya@ua.fm
\end{abstract}

The title compound, $\mathrm{C}_{27} \mathrm{H}_{20} \mathrm{~N}_{2}$, a Schiff base synthesized via a condensation reaction between anthracene-9-carbaldehyde and $N$-phenyl-p-phenylenediamine, crystallizes with three independent molecules in the asymmetric unit. The three molecules have slightly varying overall conformations, all having trans conformations with respect to the $\mathrm{C}=\mathrm{N}$ bond. In the crystal, the packing features $\mathrm{N}-\mathrm{H} \cdots \mathrm{N}$ hydrogen bonds, which connect molecules into chains extending along the $c$-axis direction, interlinked by $\mathrm{C}-\mathrm{H} \cdots \pi$ interactions (minimum $\mathrm{H} \cdots C g=2.65 \AA$ ) into sheets lying parallel to (001).

\section{Chemical context}

Anthracene derivatives have been widely used in the field of anion recognition, metal ion fluorescent sensors, as well as $p \mathrm{H}$ sensors (Gunnlaugsson et al., 2003; Chen \& Chen, 2004; Kim \& Yoon, 2002; Bernhardt et al. 2001) because of their excellent photophysical properties and high fluorescence. The crystal structures of several anthracene derivatives have been reported previously for supramolecular photochemistry (Akiba et al., 1999; Yuan et al., 2004; Yamashita et al., 2005). As part of our ongoing studies of Schiff bases (Faizi et al., 2016), we report herein on the synthesis and crystal structure of the title compound, $(E)-N^{1}$-[(anthracen-9-yl)methylidene]$\mathrm{N}^{4}$-phenylbenzene-1,4-diamine, obtained from the condensation of 9-anthracenecarboxaldehyde with $N$-phenyl- $p$ phenylenediamine.

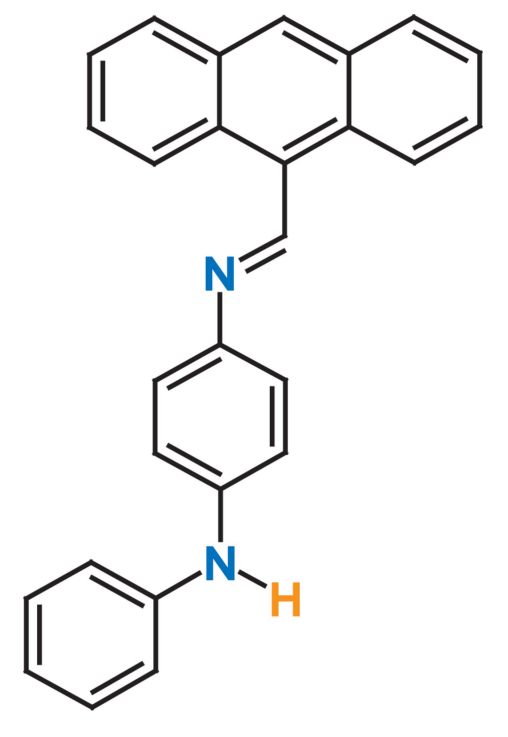




\section{Structural commentary}

The title compound, crystallizes with three independent molecules $(A, B$ and $C$ ) in the asymmetric unit (Fig. 1). Molecules $B$ and $C$ are linked by an $\mathrm{N}-\mathrm{H} \cdots \mathrm{N}$ hydrogen bond and a $\mathrm{C}-$ $\mathrm{H} \cdots \pi$ interaction, while molecule $A$ forms a $\mathrm{C}-\mathrm{H} \cdots \pi$ interaction with molecule $B$, as well as an $\mathrm{N}-\mathrm{H} \cdots \mathrm{N}$ hydrogen bond and a $\mathrm{C}-\mathrm{H} \cdots \pi$ interaction with a symmetry-generated $A$ molecule. An intramolecular $\mathrm{C}-\mathrm{H} \cdots \mathrm{N}$ hydrogen bond occurs in each molecule (Table 1). There is a slight variation (within $3 \sigma$ ) in the bond lengths and angles of the three independent molecules. All three molecules have trans conformations. The central $\mathrm{C}=\mathrm{N}(\mathrm{C} 15-\mathrm{N} 1)$ bond lengths are 1.277 (2), 1.276 (2) and 1.271 (2) ̊ for molecules $A, B$ and $C$, respectively. These are close to the literature value of $1.279 \AA$ for $\mathrm{C} s p^{2}=\mathrm{N} s p^{2}$ bonds (Fritsky et al., 2004; Penkova et al., 2010). The $\mathrm{C} 14-\mathrm{C} 15$ bond lengths between the anthracene moiety and the central $\mathrm{C}=\mathrm{N}$ bond in $A, B$ and $C$ are 1.474 (3), 1.472 (3) and 1.476 (3) $\AA$, respectively. The comparative N1$\mathrm{C} 16$ bonds connecting the central benzene ring to the central $\mathrm{C}=\mathrm{N}$ bond in $A, B$ and $C$ are $1.422(2), 1.419$ (2) and 1.420 (2) $\AA$, respectively. The $\mathrm{C} 14-\mathrm{C} 15-\mathrm{N} 1-\mathrm{C} 16$, torsion angles for the $-\mathrm{C}-\mathrm{C}-\mathrm{N}-\mathrm{C}-$ bridge groups are $-178.47(17)^{\circ}$ (for $A$ ), $-176.35(17)^{\circ}$ (for $B$ ) and $178.31(17)^{\circ}$ (for $C$ ). The comparative dihedral angles between the anthracene ring system of the molecule (defined by $\mathrm{C} 1-\mathrm{C} 14$ ) and the benzene and phenyl rings (defined by $\mathrm{C} 16-\mathrm{C} 21$ and $\mathrm{C} 22-\mathrm{C} 27)$ and between the benzene and phenyl rings, respectively, are $82.68(4), 73.76(5)$ and $25.63(11)^{\circ}$ in $A$,
Table 1

Hydrogen-bond geometry $\left(\AA{ }^{\circ}\right)$.

$C g 2, C g 4, C g 10, C g 11, C g 12, C g 13, C g 18, C g 20$ and $C g 21$ are the centroids of the $\mathrm{C} 1 A-\mathrm{C} 14 A, \mathrm{C} 16 A-\mathrm{C} 21 A, \mathrm{C} 1 B-\mathrm{C} 14 B, \mathrm{C} 8 B-\mathrm{C} 13 B, \mathrm{C} 16 B-\mathrm{C} 21 B, \mathrm{C} 22 B-\mathrm{C} 27 B$, $\mathrm{C} 1 C-\mathrm{C} 14 C, \mathrm{C} 22 \mathrm{C}-\mathrm{C} 27 \mathrm{C}$ and $\mathrm{C} 1 C-\mathrm{C} 8 C$ rings, respectively.

\begin{tabular}{lllll}
\hline$D-\mathrm{H} \cdots A$ & $D-\mathrm{H}$ & $\mathrm{H} \cdots A$ & $D \cdots A$ & $D-\mathrm{H} \cdots A$ \\
\hline $\mathrm{C} 12 A-\mathrm{H} 9 A \cdots \mathrm{N} 1 A$ & 0.93 & 2.44 & $2.989(3)$ & 118 \\
$\mathrm{C} 12 B-\mathrm{H} 9 B \cdots \mathrm{N} 1 B$ & 0.93 & 2.47 & $3.006(3)$ & 117 \\
$\mathrm{C} 2 C-\mathrm{H} 1 C \cdots \mathrm{N} 1 C$ & 0.93 & 2.51 & $3.032(3)$ & 116 \\
$\mathrm{~N} 2 A-\mathrm{H} 20 A \cdots \mathrm{N} 1 A^{\mathrm{i}}$ & $0.92(2)$ & $2.28(2)$ & $3.147(2)$ & $158.4(18)$ \\
$\mathrm{N} 2 B-\mathrm{H} 20 B \cdots \mathrm{N} 1 C^{\mathrm{ii}}$ & $0.88(2)$ & $2.19(2)$ & $3.056(2)$ & $166.2(19)$ \\
$\mathrm{N} 2 C-\mathrm{H} 20 C \cdots \mathrm{N} 1 B$ & $0.90(2)$ & $2.22(2)$ & $3.094(2)$ & $163.2(17)$ \\
$\mathrm{C} 2 A-\mathrm{H} 1 A \cdots C g 13^{\mathrm{iii}}$ & 0.93 & 2.93 & $3.7248(3)$ & 144 \\
$\mathrm{C} 2 B-\mathrm{H} 1 B \cdots C g 21^{\text {iv }}$ & 0.93 & 2.91 & $3.6227(3)$ & 134 \\
$\mathrm{C} 4 A-\mathrm{H} 3 A \cdots C g 21^{\mathrm{v}}$ & 0.93 & 2.75 & $3.6728(3)$ & 175 \\
$\mathrm{C} 10 A-\mathrm{H} 7 A \cdots C g 12^{\mathrm{ii}}$ & 0.93 & 2.80 & $3.5075(3)$ & 134 \\
$\mathrm{C} 17 A-\mathrm{H} 11 A \cdots C g 18^{\mathrm{ii}}$ & 0.93 & 2.65 & $3.5119(3)$ & 154 \\
$\mathrm{C} 17 B-\mathrm{H} 11 B \cdots C g 10^{\mathrm{v}}$ & 0.93 & 2.79 & $3.6243(3)$ & 150 \\
$\mathrm{C} 17 B-\mathrm{H} 11 B \cdots C g 11^{\mathrm{v}}$ & 0.93 & 2.89 & $3.7248(3)$ & 150 \\
$\mathrm{C} 21 C-\mathrm{H} 13 C \cdots C g 2^{\mathrm{vi}}$ & 0.93 & 2.86 & $3.7513(3)$ & 162 \\
$\mathrm{C} 23 A-\mathrm{H} 15 A \cdots C g 2^{\mathrm{i}}$ & 0.93 & 2.85 & $3.7253(3)$ & 157 \\
$\mathrm{C} 23 B-\mathrm{H} 15 B \cdots C g 11^{\mathrm{ii}}$ & 0.93 & 2.87 & $3.7057(3)$ & 149 \\
$\mathrm{C} 25 A-\mathrm{H} 17 A \cdots C g 20^{\text {vii }}$ & 0.93 & 2.96 & $3.6262(3)$ & 130 \\
$\mathrm{C} 25 B-\mathrm{H} 17 B \cdots C g 4^{\mathrm{viii}}$ & 0.93 & 3.00 & $3.6476(3)$ & 128 \\
$\mathrm{C} 25 C-\mathrm{H} 17 C \cdots C g 12^{\text {iv }}$ & 0.93 & 2.84 & $3.6263(3)$ & 143 \\
\hline
\end{tabular}

Symmetry codes: (i) $x,-y+\frac{3}{2}, z-\frac{1}{2}$; (ii) $x, y, z+1$; (iii) $x-1, y, z-1$; (iv) $-x+2,-y+1,-z+1 ; \quad$ (v) $-x+1,-y+1,-z+1 ; \quad$ (vi) $\quad x, y, z-1$; (vii) $x-1,-y+\frac{3}{2}, z+\frac{1}{2}$; (viii) $x+1, y, z$.

$80.10(4), 78.82(5)$ and $22.56(11)^{\circ}$ (in $B$ ) and $85.02(5)$, 81.66 (5) and $16.25(11)^{\circ}$ (in $C$ ).

Figure 1

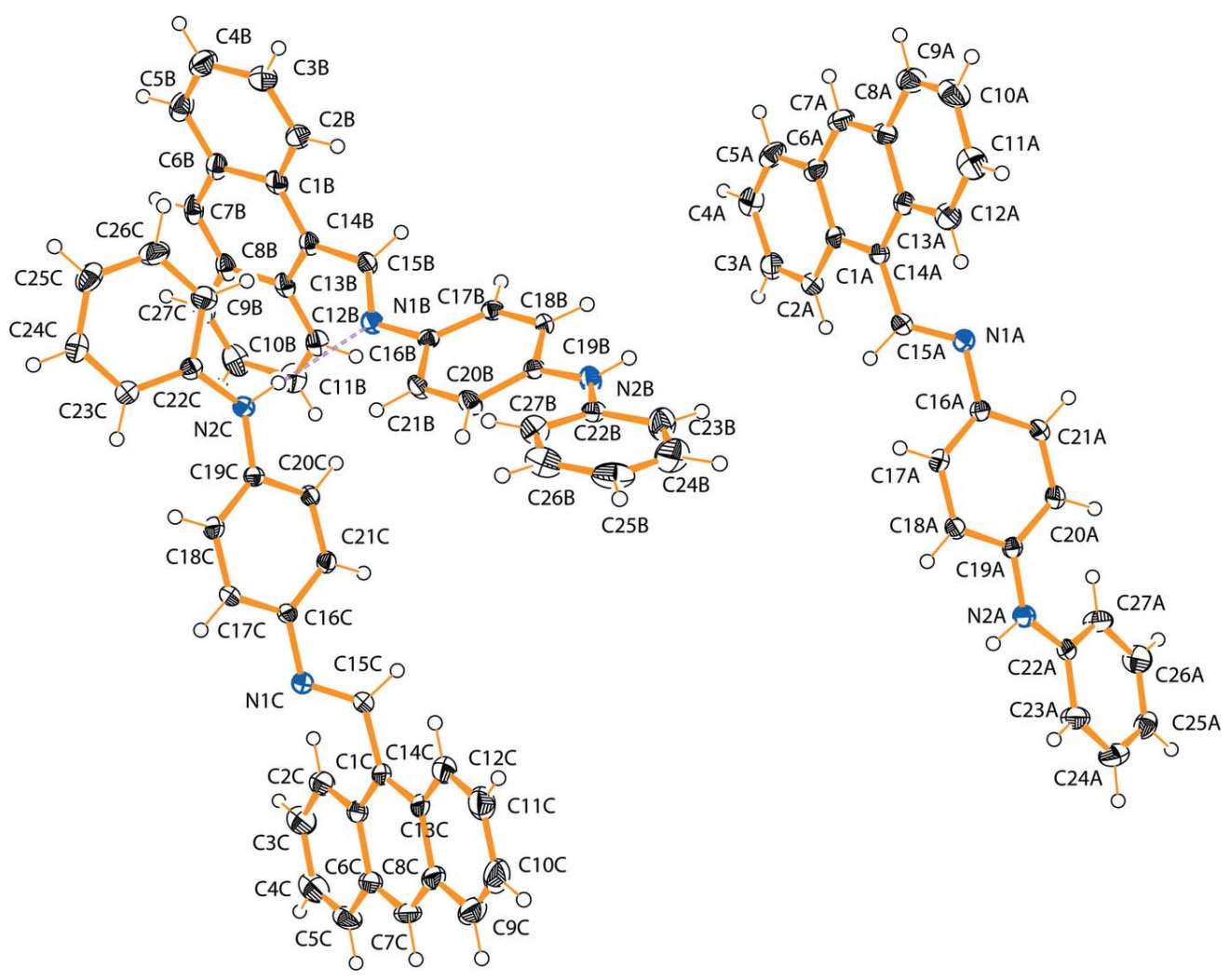

A view of the three independent molecules $(A, B$ and $C)$ in the asymmetric unit of the title compound with the atom-labelling scheme and $40 \%$ probability displacement ellipsoids, showing the $\mathrm{C}-\mathrm{H} \cdots \mathrm{N}$ interaction between molecules $B$ and $C$ as a dashed line. 


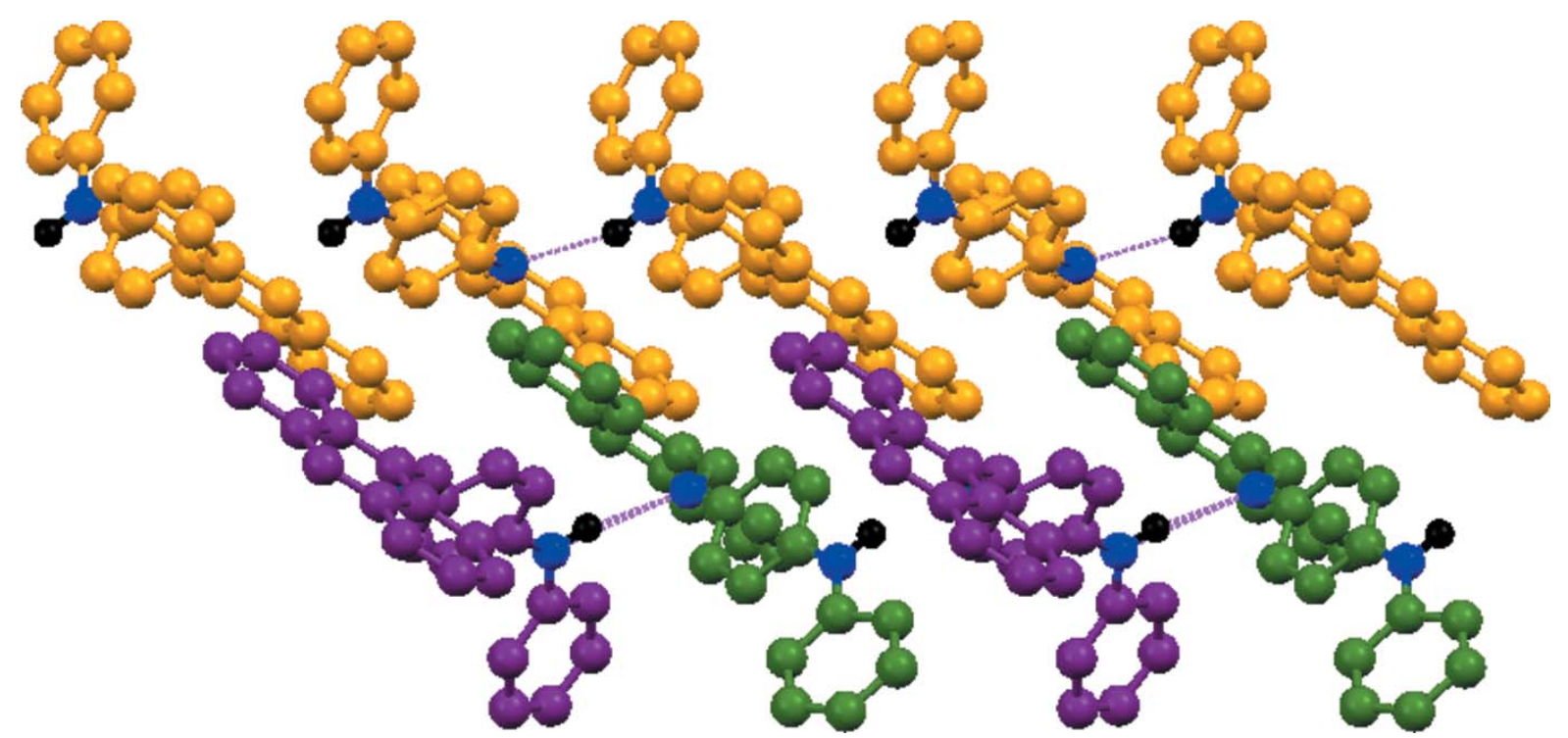

Figure 2

A view of the hydrogen-bonded chains propagating in [001]. Hydrogen bonds are shown as dashed lines; see Table 1 for details.

\section{Supramolecular features}

In the crystal, the molecules are connected by $\mathrm{N}-\mathrm{H} \cdots \mathrm{N}$ hydrogen bonds that result in separate $-A-A-A-A$ - and $-B-$ $C-B-C$ - chains, which both propagate in [001] (Table 1 and Fig. 2). The chains are linked via $\mathrm{C}-\mathrm{H} \cdots \pi$ interactions between the phenyl and central benzene rings and those of the anthracene moiety groups of neighbouring molecules [minimum $\mathrm{C} 17 \mathrm{~A}-\mathrm{H} \cdots \mathrm{Cg}(\mathrm{C} 1 C-\mathrm{C} 14 C)=2.65 \AA$ 年 $\mathrm{C}-\mathrm{H} \cdots C g$ $=154^{\circ}$, forming layers lying parallel to (001) (Fig. 3, Table 1).

\section{Database survey}

A search of the Cambridge Structural Database (Version 5.36; last update November 2014; Groom et al., 2016) gave three hits for Schiff base compounds involving $N$-phenyl- $p$-phenyl- enediamine. Of these three compounds, N1-phenyl- $N-4-$ (quinolin-2-ylmethylene)benzene-1,4-diamine \{synonym: $\mathrm{N}$ phenyl-4-[(quinolin-2-ylmethylene)amino]-aniline; WOJJIQ (Faizi et al., 2014] is the most similar to the title compound, with dihedral angles between quinoline ring system (r.m.s. deviation $=0.027 \AA$ ) and the central benzene and terminal phenyl rings of $44.72(7)$ and $9.02(4)^{\circ}$, respectively. Another similar structure crystal is that of N1-phenyl- $N 4-[(E)$-(pyren1-yl)-methylidene]benzene-1,4-diamine (Faizi \& Prisyazhnaya, 2015), which has dihedral angles between the pyrenyl ring system (r.m.s. deviation $=0.027 \AA$ ) and the central and terminal benzene rings of $43.43(9)$ and $29.33(7)^{\circ}$, respectively. Some similar ligands have been used as dual chemosensors for the detection of $\mathrm{Cu}^{2+}$ and $\mathrm{Hg}^{2+}$ ions (Udhayakumari \& Velmathi, 2015) but their crystal structures have not been reported.

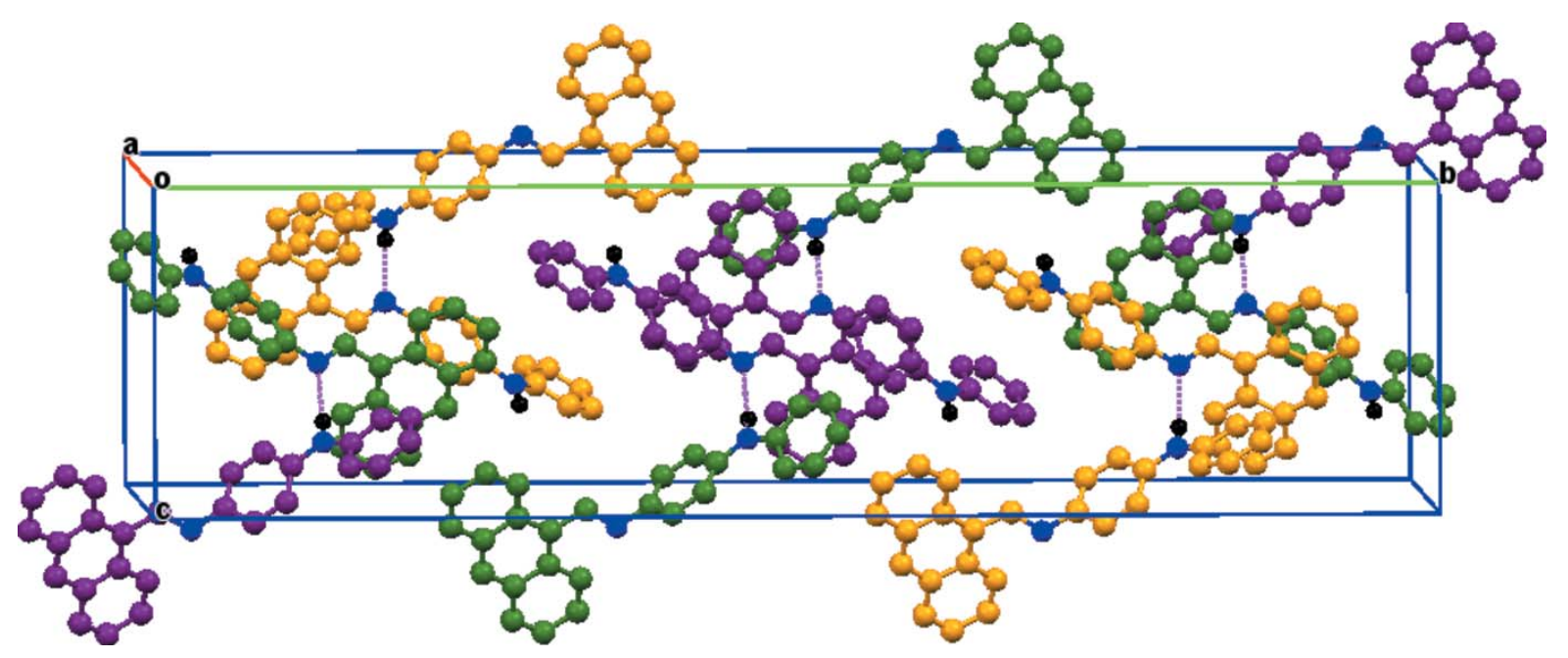

Figure 3

A view along the $a$ axis of the crystal packing of the title compound. The hydrogen bonds are shown as dashed lines. 


\section{Synthesis and crystallization}

$80 \mathrm{mg}(0.435 \mathrm{mmol})$ of $N$-phenyl-p-phenylenediamine were dissolved in $10 \mathrm{ml}$ of absolute ethanol. To this solution, $89 \mathrm{mg}$ $(0.434 \mathrm{mmol})$ of 9 -anthracenecarboxaldehyde in $5 \mathrm{ml}$ of absolute ethanol was added dropwise under stirring. The mixture was stirred for $10 \mathrm{~min}$, two drops of glacial acetic acid were then added and the mixture was further refluxed for $2 \mathrm{~h}$. The resulting yellow precipitate was recovered by filtration, washed several times with small portions of ice-cold ethanol and then with diethyl ether to give $140 \mathrm{mg}$ (87\%) of the title compound. Dark-yellow block-like crystals suitable for X-ray analysis were obtained within 3 days by slow evaporation of a solution in $\mathrm{MeOH}$.

\section{Refinement}

Crystal data, data collection and structure refinement details are summarized in Table 2. The $\mathrm{N}$-bound $\mathrm{H}$ atoms were located in a difference Fourier map. Their positional and isotropic thermal parameters were included in further stages of the refinement. All $\mathrm{C}$-bound $\mathrm{H}$ atoms were positioned geometrically and refined using a riding model with $\mathrm{C}-\mathrm{H}=$ $0.93-0.97 \AA$ and with $U_{\text {iso }}(\mathrm{H})=1.2-1.5 U_{\text {eq }}(\mathrm{C})$.

\section{Acknowledgements}

The authors are grateful to the National Taras Shevchenko University, Department of Chemistry, Volodymyrska str. 64, 01601 Kyiv, Ukraine, for financial support and Dr Igor Fritsky for helpful discussions.

\section{References}

Akiba, K., Yamashita, M., Yamamoto, Y. \& Nagase, S. (1999). J. Am. Chem. Soc. 121, 10644-10645.

Altomare, A., Burla, M. C., Camalli, M., Cascarano, G. L., Giacovazzo, C., Guagliardi, A., Moliterni, A. G. G., Polidori, G. \& Spagna, R. (1999). J. Appl. Cryst. 32, 115-119.

Bernhardt, P. V., Moore, E. G. \& Riley, M. J. (2001). Inorg. Chem. 40, 5799-5805.

Brandenberg, K. \& Putz, H. (2006). DIAMOND. Crystal Impact GbR, Bonn, Germany.

Bruker (2003). SMART, SAINT and SADABS. Bruker AXS Inc., Madison, Wisconsin, USA.

Chen, Q. Y. \& Chen, C. F. (2004). Tetrahedron Lett. 45, 6493-6496.

Faizi, M. S. H., Gupta, S., Mohan, V. K., Jain, K. V. \& Sen, P. (2016). Sens. Actuators B Chem. 222, 15-20.
Table 2

Experimental details.

\begin{tabular}{|c|c|}
\hline \multicolumn{2}{|l|}{ Crystal data } \\
\hline Chemical formula & $\mathrm{C}_{27} \mathrm{H}_{20} \mathrm{~N}_{2}$ \\
\hline$M_{\mathrm{r}}$ & 372.45 \\
\hline Crystal system, space group & Monoclinic, $P 2_{1} / c$ \\
\hline Temperature $(\mathrm{K})$ & 100 \\
\hline$a, b, c(\AA)$ & 11.1554 (8), 45.224 (3), $11.5856(8)$ \\
\hline$\beta\left({ }^{\circ}\right)$ & $96.645(2)$ \\
\hline$V\left(\mathrm{~A}^{3}\right)$ & $5805.5(7)$ \\
\hline$Z$ & 12 \\
\hline Radiation type & Мo $K \alpha$ \\
\hline$\mu\left(\mathrm{mm}^{-1}\right)$ & 0.08 \\
\hline Crystal size $(\mathrm{mm})$ & $0.20 \times 0.15 \times 0.10$ \\
\hline \multicolumn{2}{|l|}{ Data collection } \\
\hline Diffractometer & BRUKER SMART APEX CCD \\
\hline Absorption correction & $\begin{array}{l}\text { Multi-scan (SADABS; Bruker, } \\
\text { 2003) }\end{array}$ \\
\hline$T_{\min }, T_{\max }$ & $0.944,0.981$ \\
\hline $\begin{array}{l}\text { No. of measured, independent and } \\
\text { observed }[I>2 \sigma(I)] \text { reflections }\end{array}$ & 70261, 10276, 6957 \\
\hline$R_{\text {int }}$ & 0.066 \\
\hline$(\sin \theta / \lambda)_{\max }\left(\AA^{-1}\right)$ & 0.596 \\
\hline \multicolumn{2}{|l|}{ Refinement } \\
\hline$R\left[F^{2}>2 \sigma\left(F^{2}\right)\right], w R\left(F^{2}\right), S$ & $0.052,0.121,1.04$ \\
\hline No. of reflections & 10276 \\
\hline No. of parameters & 796 \\
\hline H-atom treatment & $\begin{array}{l}\mathrm{H} \text { atoms treated by a mixture of } \\
\text { independent and constrained } \\
\text { refinement }\end{array}$ \\
\hline$\Delta \rho_{\max }, \Delta \rho_{\min }\left(\mathrm{e} \AA^{-3}\right)$ & $0.18,-0.28$ \\
\hline
\end{tabular}

Computer programs: SMART and SAINT (Bruker, 2003), SIR97 (Altomare et al., 1999), SHELXL2014 (Sheldrick, 2015) and DIAMOND (Brandenberg \& Putz, 2006).

Faizi, M. S. H., Mashrai, A., Garandal, S. \& Shahid, M. (2014). Acta Cryst. E70, o905-o906.

Faizi, M. S. H. \& Prisyazhnaya, E. V. (2015). Acta Cryst. E71, 261-263.

Fritsky, I. O., Świątek-Kozłowska, J., Dobosz, A., Sliva, T. Y. \& Dudarenko, N. M. (2004). Inorg. Chim. Acta, 357, 3746-3752.

Groom, C. R., Bruno, I. J., Lightfoot, M. P. \& Ward, S. C. (2016). Acta Cryst. B72, 171-179.

Gunnlaugsson, T., Lee, T. C. \& Parkesh, R. (2003). Org. Lett. 5, 40654068.

Kim, S. K. \& Yoon, J. (2002). Chem. Commun. pp. 770-771.

Penkova, L., Demeshko, S., Pavlenko, V. A., Dechert, S., Meyer, F. \& Fritsky, I. O. (2010). Inorg. Chim. Acta, 363, 3036-3040.

Sheldrick, G. M. (2015). Acta Cryst. C71, 3-8.

Udhayakumari, D. \& Velmathi, S. (2015). Ind. Eng. Chem. Res. 54, 3541-3547.

Yamashita, M., Yamamoto, Y., Akiba, K., Hashizume, D., Iwasaki, F., Takagi, N. \& Nagase, S. (2005). J. Am. Chem. Soc. 127, 4354-4371. Yuan, W.-B., Yan, L. \& Yang, R.-D. (2004). Acta Cryst. E60, o2447o2448. 


\section{supporting information}

Acta Cryst. (2017). E73, 137-140 [https://doi.org/10.1107/S2056989016020612]

Crystal structure of $(E)-N^{1}-\left[(\right.$ anthracen-9-yl)methylidene $]-N^{4}$-phenylbenzene-1,4-diamine

Md. Serajul Haque Faizi, Ashanul Haque, Musheer Ahmad and Irina A. Golenya

Computing details

Data collection: SMART (Bruker, 2003); cell refinement: SAINT (Bruker, 2003); data reduction: SAINT (Bruker, 2003); program(s) used to solve structure: SIR97 (Altomare et al., 1999); program(s) used to refine structure: SHELXL2014 (Sheldrick, 2015); molecular graphics: DIAMOND (Brandenberg \& Putz, 2006); software used to prepare material for publication: DIAMOND (Brandenberg \& Putz, 2006).

(E)-N $N^{1}$-[(Anthracen-9-yl)methylidene]- $N^{4}$-phenylbenzene-1,4-diamine

Crystal data

$\mathrm{C}_{27} \mathrm{H}_{20} \mathrm{~N}_{2}$

$M_{r}=372.45$

Monoclinic, $P 2_{1} / c$

$a=11.1554(8) \AA$

$b=45.224(3) \AA$

$c=11.5856(8) \AA$

$\beta=96.645(2)^{\circ}$

$V=5805.5(7) \AA^{3}$

$Z=12$

\section{Data collection}

BRUKER SMART APEX CCD diffractometer

Radiation source: fine-focus sealed tube Graphite monochromator $\omega$ scans

Absorption correction: multi-scan

(SADABS; Bruker, 2003)

$T_{\min }=0.944, T_{\max }=0.981$

Refinement

Refinement on $F^{2}$

Least-squares matrix: full

$R\left[F^{2}>2 \sigma\left(F^{2}\right)\right]=0.052$

$w R\left(F^{2}\right)=0.121$

$S=1.04$

10276 reflections

796 parameters

0 restraints
$F(000)=2352$

$D_{\mathrm{x}}=1.278 \mathrm{Mg} \mathrm{m}^{-3}$

Mo $K \alpha$ radiation, $\lambda=0.71073 \AA$

Cell parameters from 9870 reflections

$\theta=2.7-27.3^{\circ}$

$\mu=0.08 \mathrm{~mm}^{-1}$

$T=100 \mathrm{~K}$

Block, yellow

$0.20 \times 0.15 \times 0.10 \mathrm{~mm}$

70261 measured reflections

10276 independent reflections 6957 reflections with $I>2 \sigma(I)$

$R_{\text {int }}=0.066$

$\theta_{\max }=25.1^{\circ}, \theta_{\min }=2.1^{\circ}$

$h=-13 \rightarrow 13$

$k=-53 \rightarrow 53$

$l=-13 \rightarrow 13$

Hydrogen site location: mixed

$\mathrm{H}$ atoms treated by a mixture of independent and constrained refinement

$w=1 /\left[\sigma^{2}\left(F_{0}^{2}\right)+(0.0468 P)^{2}+1.868 P\right]$

where $P=\left(F_{\mathrm{o}}^{2}+2 F_{\mathrm{c}}^{2}\right) / 3$

$(\Delta / \sigma)_{\max }=0.001$

$\Delta \rho_{\max }=0.18 \mathrm{e} \AA^{-3}$

$\Delta \rho_{\min }=-0.28$ e $\AA^{-3}$ 


\section{Special details}

Geometry. All esds (except the esd in the dihedral angle between two 1.s. planes) are estimated using the full covariance matrix. The cell esds are taken into account individually in the estimation of esds in distances, angles and torsion angles; correlations between esds in cell parameters are only used when they are defined by crystal symmetry. An approximate (isotropic) treatment of cell esds is used for estimating esds involving l.s. planes.

Fractional atomic coordinates and isotropic or equivalent isotropic displacement parameters $\left(\AA^{2}\right)$

\begin{tabular}{|c|c|c|c|c|}
\hline & $x$ & $y$ & $z$ & $U_{\text {iso }} * / U_{\text {eq }}$ \\
\hline $\mathrm{C} 1 \mathrm{~A}$ & $0.26112(17)$ & $0.61937(4)$ & $1.02008(17)$ & $0.0262(5)$ \\
\hline C1B & $0.71492(18)$ & $0.45788(4)$ & $0.46717(17)$ & $0.0282(5)$ \\
\hline $\mathrm{C} 1 \mathrm{C}$ & $0.55519(18)$ & $0.68363(4)$ & $-0.18917(18)$ & $0.0314(5)$ \\
\hline $\mathrm{C} 2 \mathrm{~A}$ & $0.18402(18)$ & $0.62145(5)$ & $0.91327(17)$ & $0.0313(5)$ \\
\hline H1A & 0.1713 & 0.6398 & 0.8776 & $0.038^{*}$ \\
\hline $\mathrm{C} 2 \mathrm{~B}$ & $0.78872(19)$ & $0.45822(4)$ & $0.57631(18)$ & $0.0327(5)$ \\
\hline H1B & 0.7929 & 0.4753 & 0.6211 & $0.039 *$ \\
\hline $\mathrm{C} 2 \mathrm{C}$ & $0.49613(18)$ & $0.65789(5)$ & $-0.23828(18)$ & $0.0357(5)$ \\
\hline $\mathrm{H} 1 \mathrm{C}$ & 0.5033 & 0.6402 & -0.1971 & $0.043^{*}$ \\
\hline $\mathrm{C} 3 \mathrm{~A}$ & $0.12888(19)$ & $0.59724(5)$ & $0.86268(19)$ & $0.0352(5)$ \\
\hline $\mathrm{H} 2 \mathrm{~A}$ & 0.0798 & 0.5993 & 0.7925 & $0.042 *$ \\
\hline C3B & $0.8530(2)$ & $0.43405(5)$ & $0.61612(19)$ & $0.0393(6)$ \\
\hline $\mathrm{H} 2 \mathrm{~B}$ & 0.8994 & 0.4348 & 0.6881 & $0.047^{*}$ \\
\hline $\mathrm{C} 3 \mathrm{C}$ & $0.4297(2)$ & $0.65861(6)$ & $-0.34359(19)$ & $0.0442(6)$ \\
\hline $\mathrm{H} 2 \mathrm{C}$ & 0.3931 & 0.6413 & -0.3737 & $0.053 *$ \\
\hline $\mathrm{C} 4 \mathrm{~A}$ & $0.14451(19)$ & $0.56901(5)$ & $0.9145(2)$ & $0.0380(6)$ \\
\hline $\mathrm{H} 3 \mathrm{~A}$ & 0.1077 & 0.5525 & 0.8779 & $0.046^{*}$ \\
\hline C4B & $0.8504(2)$ & $0.40788(5)$ & $0.5497(2)$ & $0.0418(6)$ \\
\hline H3B & 0.8948 & 0.3915 & 0.5779 & $0.050 *$ \\
\hline $\mathrm{C} 4 \mathrm{C}$ & $0.4149(2)$ & $0.68491(6)$ & $-0.4086(2)$ & $0.0503(7)$ \\
\hline $\mathrm{H} 3 \mathrm{C}$ & 0.3687 & 0.6850 & -0.4808 & $0.060 *$ \\
\hline $\mathrm{C} 5 \mathrm{~A}$ & $0.21301(19)$ & $0.56617(5)$ & $1.0172(2)$ & $0.0375(6)$ \\
\hline $\mathrm{H} 4 \mathrm{~A}$ & 0.2215 & 0.5476 & 1.0519 & $0.045^{*}$ \\
\hline C5B & $0.7831(2)$ & $0.40671(5)$ & $0.4451(2)$ & $0.0385(6)$ \\
\hline $\mathrm{H} 4 \mathrm{~B}$ & 0.7823 & 0.3894 & 0.4017 & $0.046^{*}$ \\
\hline $\mathrm{C} 5 \mathrm{C}$ & $0.4680(2)$ & $0.71001(6)$ & $-0.3653(2)$ & $0.0474(7)$ \\
\hline $\mathrm{H} 4 \mathrm{C}$ & 0.4576 & 0.7274 & -0.4083 & $0.057^{*}$ \\
\hline C6A & $0.27303(18)$ & $0.59094(4)$ & $1.07454(18)$ & $0.0309(5)$ \\
\hline C6B & $0.71353(18)$ & $0.43128(4)$ & $0.39983(18)$ & $0.0303(5)$ \\
\hline C6C & $0.53946(19)$ & $0.71046(5)$ & -0.25565 (19) & $0.0366(6)$ \\
\hline C7A & $0.33961(19)$ & $0.58833(5)$ & $1.18216(19)$ & $0.0351(5)$ \\
\hline $\mathrm{H} 5 \mathrm{~A}$ & 0.3449 & 0.5699 & 1.2181 & $0.042 *$ \\
\hline C7B & $0.64679(18)$ & $0.43032(4)$ & $0.29127(18)$ & $0.0331(5)$ \\
\hline H5B & 0.6468 & 0.4130 & 0.2481 & $0.040^{*}$ \\
\hline $\mathrm{C} 7 \mathrm{C}$ & $0.5933(2)$ & $0.73624(5)$ & $-0.2093(2)$ & $0.0435(6)$ \\
\hline $\mathrm{H} 5 \mathrm{C}$ & 0.5837 & 0.7535 & -0.2528 & $0.052 *$ \\
\hline C8A & $0.39895(18)$ & $0.61213(5)$ & $1.23881(18)$ & $0.0318(5)$ \\
\hline $\mathrm{C} 8 \mathrm{~B}$ & $0.57997(18)$ & $0.45425(4)$ & $0.24448(17)$ & $0.0298(5)$ \\
\hline $\mathrm{C} 8 \mathrm{C}$ & $0.6606(2)$ & $0.73722(5)$ & $-0.1012(2)$ & $0.0392(6)$ \\
\hline
\end{tabular}




\begin{tabular}{|c|c|c|c|c|}
\hline C9A & $0.4676(2)$ & $0.60887(5)$ & $1.34924(19)$ & $0.0416(6)$ \\
\hline H6A & 0.4707 & 0.5905 & 1.3858 & $0.050 *$ \\
\hline C9B & $0.5082(2)$ & $0.45245(5)$ & $0.13519(18)$ & $0.0382(6)$ \\
\hline H6B & 0.5086 & 0.4351 & 0.0922 & $0.046^{*}$ \\
\hline $\mathrm{C} 9 \mathrm{C}$ & $0.7169(2)$ & $0.76360(5)$ & $-0.0555(3)$ & $0.0531(7)$ \\
\hline $\mathrm{H} 6 \mathrm{C}$ & 0.7056 & 0.7810 & -0.0983 & $0.064 *$ \\
\hline $\mathrm{C} 10 \mathrm{~A}$ & $0.5281(2)$ & $0.63167(6)$ & $1.4019(2)$ & $0.0467(6)$ \\
\hline H7A & 0.5723 & 0.6291 & 1.4743 & $0.056^{*}$ \\
\hline $\mathrm{C} 10 \mathrm{~B}$ & $0.4397(2)$ & $0.47522(5)$ & $0.09266(19)$ & $0.0431(6)$ \\
\hline H7B & 0.3930 & 0.4736 & 0.0211 & $0.052 *$ \\
\hline $\mathrm{C} 10 \mathrm{C}$ & $0.7862(3)$ & $0.76430(5)$ & $0.0479(3)$ & $0.0566(8)$ \\
\hline $\mathrm{H} 7 \mathrm{C}$ & 0.8209 & 0.7820 & 0.0760 & $0.068 *$ \\
\hline C11A & $0.52493(19)$ & $0.65969(5)$ & $1.34739(19)$ & $0.0428(6)$ \\
\hline H8A & 0.5686 & 0.6753 & 1.3838 & $0.051 *$ \\
\hline C11B & $0.4388(2)$ & $0.50170(5)$ & $0.15699(19)$ & $0.0408(6)$ \\
\hline H8B & 0.3904 & 0.5173 & 0.1275 & $0.049 *$ \\
\hline $\mathrm{C} 11 \mathrm{C}$ & $0.8057(2)$ & $0.73793(5)$ & $0.1131(2)$ & $0.0501(7)$ \\
\hline $\mathrm{H} 8 \mathrm{C}$ & 0.8543 & 0.7383 & 0.1839 & $0.060 *$ \\
\hline C12A & $0.45909(18)$ & $0.66419(5)$ & $1.24274(18)$ & $0.0339(5)$ \\
\hline H9A & 0.4579 & 0.6828 & 1.2088 & $0.041 *$ \\
\hline $\mathrm{C} 12 \mathrm{~B}$ & $0.50720(18)$ & $0.50484(5)$ & $0.26098(18)$ & $0.0314(5)$ \\
\hline H9B & 0.5058 & 0.5227 & 0.3009 & $0.038 *$ \\
\hline $\mathrm{C} 12 \mathrm{C}$ & $0.7541(2)$ & $0.71209(5)$ & $0.07366(19)$ & $0.0409(6)$ \\
\hline $\mathrm{H} 9 \mathrm{C}$ & 0.7688 & 0.6950 & 0.1177 & $0.049 *$ \\
\hline $\mathrm{C} 13 \mathrm{~A}$ & $0.39169(17)$ & $0.64070(4)$ & $1.18408(17)$ & $0.0271(5)$ \\
\hline C13B & 0.58098 (17) & $0.48128(4)$ & $0.30983(17)$ & $0.0266(5)$ \\
\hline $\mathrm{C} 13 \mathrm{C}$ & $0.67808(19)$ & $0.71060(4)$ & $-0.03370(18)$ & $0.0328(5)$ \\
\hline $\mathrm{C} 14 \mathrm{~A}$ & $0.32106(17)$ & 0.64399 (4) & $1.07609(16)$ & $0.0252(5)$ \\
\hline C14B & $0.65076(17)$ & 0.48293 (4) & $0.41958(17)$ & $0.0249(5)$ \\
\hline $\mathrm{C} 14 \mathrm{C}$ & $0.62503(18)$ & 0.68413 (4) & $-0.07964(18)$ & $0.0298(5)$ \\
\hline $\mathrm{C} 15 \mathrm{~A}$ & 0.30094 (17) & $0.67319(4)$ & $1.02085(17)$ & $0.0262(5)$ \\
\hline $\mathrm{H} 10 \mathrm{~A}$ & 0.3043 & 0.6748 & 0.9412 & $0.031 *$ \\
\hline $\mathrm{C} 15 \mathrm{~B}$ & $0.66532(17)$ & 0.51095 (4) & $0.48428(16)$ & $0.0248(5)$ \\
\hline $\mathrm{H} 10 \mathrm{~B}$ & 0.6577 & 0.5111 & 0.5634 & $0.030 *$ \\
\hline $\mathrm{C} 15 \mathrm{C}$ & $0.65310(18)$ & 0.65659 (4) & $-0.01366(17)$ & $0.0275(5)$ \\
\hline $\mathrm{H} 10 \mathrm{C}$ & 0.6407 & 0.6557 & 0.0643 & $0.033^{*}$ \\
\hline C16A & $0.25826(17)$ & $0.72330(4)$ & $1.01442(16)$ & $0.0233(4)$ \\
\hline C16B & $0.70840(16)$ & $0.56121(4)$ & $0.50147(15)$ & $0.0211(4)$ \\
\hline $\mathrm{C} 16 \mathrm{C}$ & $0.72269(17)$ & 0.60827 (4) & $0.00646(15)$ & $0.0215(4)$ \\
\hline C17A & $0.32184(17)$ & $0.73145(4)$ & $0.92280(16)$ & $0.0244(4)$ \\
\hline H11A & 0.3854 & 0.7197 & 0.9041 & $0.029 *$ \\
\hline C17B & $0.65393(16)$ & $0.56740(4)$ & $0.60083(15)$ & 0.0225 \\
\hline H11B & 0.5946 & 0.5548 & 0.6227 & $0.027^{*}$ \\
\hline $\mathrm{C} 17 \mathrm{C}$ & $0.82786(18)$ & $0.59356(4)$ & $-0.00799(16)$ & $0.0267(5)$ \\
\hline $\mathrm{H} 11 \mathrm{C}$ & 0.8743 & 0.5999 & -0.0649 & $0.032 *$ \\
\hline C18A & $0.29143(17)$ & $0.75671(4)$ & $0.85951(16)$ & $0.0240(4)$ \\
\hline $\mathrm{H} 12 \mathrm{~A}$ & 0.3351 & 0.7619 & 0.7988 & $0.029 *$ \\
\hline C18B & $0.68698(16)$ & $0.59196(4)$ & $0.66704(16)$ & $0.0226(4)$ \\
\hline
\end{tabular}




\begin{tabular}{|c|c|c|c|c|}
\hline H12B & 0.6498 & 0.5956 & 0.7334 & $0.027^{*}$ \\
\hline $\mathrm{C} 18 \mathrm{C}$ & $0.86612(17)$ & $0.56949(4)$ & $0.06038(16)$ & $0.0266(5)$ \\
\hline $\mathrm{H} 12 \mathrm{C}$ & 0.9396 & 0.5606 & 0.0515 & $0.032 *$ \\
\hline $\mathrm{C} 19 \mathrm{~A}$ & $0.19624(17)$ & $0.77470(4)$ & 0.88491 (16) & $0.0220(4)$ \\
\hline C19B & $0.77522(16)$ & $0.61147(4)$ & $0.63675(15)$ & $0.0210(4)$ \\
\hline $\mathrm{C} 19 \mathrm{C}$ & $0.79554(17)$ & $0.55846(4)$ & $0.14235(15)$ & $0.0211(4)$ \\
\hline C20A & $0.13906(18)$ & $0.76750(4)$ & $0.98216(16)$ & $0.0289(5)$ \\
\hline H13A & 0.0791 & 0.7798 & 1.0045 & $0.035^{*}$ \\
\hline C20B & $0.82151(18)$ & $0.60648(4)$ & $0.53181(16)$ & $0.0287(5)$ \\
\hline H13B & 0.8753 & 0.6200 & 0.5058 & $0.034 *$ \\
\hline $\mathrm{C} 20 \mathrm{C}$ & 0.68619 (17) & $0.57274(4)$ & $0.15263(16)$ & $0.0231(4)$ \\
\hline $\mathrm{H} 14 \mathrm{C}$ & 0.6362 & 0.5655 & 0.2050 & $0.028^{*}$ \\
\hline $\mathrm{C} 21 \mathrm{~A}$ & $0.17071(18)$ & $0.74221(4)$ & $1.04569(16)$ & $0.0287(5)$ \\
\hline H14A & 0.1322 & 0.7379 & 1.1107 & $0.034 *$ \\
\hline $\mathrm{C} 21 \mathrm{~B}$ & $0.78838(18)$ & $0.58171(4)$ & $0.46604(16)$ & $0.0278(5)$ \\
\hline H14B & 0.8206 & 0.5788 & 0.3964 & $0.033^{*}$ \\
\hline $\mathrm{C} 21 \mathrm{C}$ & $0.65063(17)$ & $0.59727(4)$ & $0.08713(15)$ & $0.0236(4)$ \\
\hline H13C & 0.5781 & 0.6066 & 0.0968 & $0.028 *$ \\
\hline $\mathrm{C} 22 \mathrm{~A}$ & $0.06858(17)$ & $0.81786(4)$ & $0.80373(16)$ & $0.0238(4)$ \\
\hline $\mathrm{C} 22 \mathrm{~B}$ & $0.90242(17)$ & $0.65480(4)$ & $0.71494(16)$ & $0.0238(4)$ \\
\hline $\mathrm{C} 22 \mathrm{C}$ & $0.92058(17)$ & $0.51468(4)$ & $0.22658(16)$ & $0.0235(4)$ \\
\hline $\mathrm{C} 23 \mathrm{~A}$ & $0.0768(2)$ & $0.84370(5)$ & 0.74049 (19) & $0.0386(6)$ \\
\hline H15A & 0.1485 & 0.8480 & 0.7103 & $0.046^{*}$ \\
\hline C23B & $0.8996(2)$ & $0.67904(5)$ & $0.78831(19)$ & $0.0408(6)$ \\
\hline H15B & 0.8339 & 0.6816 & 0.8298 & $0.049^{*}$ \\
\hline $\mathrm{C} 23 \mathrm{C}$ & $1.00215(18)$ & $0.51024(4)$ & $0.14642(18)$ & $0.0321(5)$ \\
\hline $\mathrm{H} 15 \mathrm{C}$ & 0.9970 & 0.5217 & 0.0795 & $0.038^{*}$ \\
\hline $\mathrm{C} 24 \mathrm{~A}$ & $-0.0180(2)$ & $0.86307(5)$ & $0.7212(2)$ & $0.0432(6)$ \\
\hline H16A & -0.0101 & 0.8800 & 0.6773 & $0.052 *$ \\
\hline $\mathrm{C} 24 \mathrm{~B}$ & $0.9923(2)$ & $0.69923(5)$ & $0.8007(2)$ & $0.0508(7)$ \\
\hline H16B & 0.9884 & 0.7152 & 0.8506 & $0.061^{*}$ \\
\hline $\mathrm{C} 24 \mathrm{C}$ & $1.09106(19)$ & $0.48884(4)$ & $0.16520(18)$ & $0.0346(5)$ \\
\hline $\mathrm{H} 16 \mathrm{C}$ & 1.1460 & 0.4865 & 0.1113 & $0.042 *$ \\
\hline $\mathrm{C} 25 \mathrm{~A}$ & $-0.1242(2)$ & $0.85767(5)$ & $0.76608(18)$ & $0.0357(5)$ \\
\hline H17A & -0.1883 & 0.8709 & 0.7543 & $0.043^{*}$ \\
\hline $\mathrm{C} 25 \mathrm{~B}$ & $1.0897(2)$ & $0.69612(5)$ & $0.7408(2)$ & $0.0461(6)$ \\
\hline H17B & 1.1516 & 0.7100 & 0.7483 & $0.055^{*}$ \\
\hline $\mathrm{C} 25 \mathrm{C}$ & 1.09985 (19) & $0.47100(5)$ & $0.26146(19)$ & $0.0375(6)$ \\
\hline $\mathrm{H} 17 \mathrm{C}$ & 1.1585 & 0.4563 & 0.2721 & $0.045^{*}$ \\
\hline $\mathrm{C} 26 \mathrm{~A}$ & $-0.1336(2)$ & $0.83227(5)$ & $0.8286(2)$ & $0.0419(6)$ \\
\hline $\mathrm{H} 18 \mathrm{~A}$ & -0.2051 & 0.8284 & 0.8599 & $0.050^{*}$ \\
\hline $\mathrm{C} 26 \mathrm{~B}$ & 1.0947 (2) & $0.67210(5)$ & $0.66918(19)$ & $0.0427(6)$ \\
\hline H18B & 1.1611 & 0.6697 & 0.6284 & $0.051^{*}$ \\
\hline $\mathrm{C} 26 \mathrm{C}$ & $1.0197(2)$ & $0.47527(5)$ & $0.34235(19)$ & $0.0402(6)$ \\
\hline $\mathrm{H} 18 \mathrm{C}$ & 1.0246 & 0.4634 & 0.4084 & $0.048^{*}$ \\
\hline $\mathrm{C} 27 \mathrm{~A}$ & $-0.03963(19)$ & $0.81222(5)$ & $0.84664(18)$ & $0.0361(5)$ \\
\hline H19A & -0.0493 & 0.7949 & 0.8878 & $0.043 *$ \\
\hline $\mathrm{C} 27 \mathrm{~B}$ & $1.00284(18)$ & $0.65145(5)$ & $0.65651(18)$ & $0.0331(5)$ \\
\hline
\end{tabular}




$\begin{array}{lllll}\text { H19B } & 1.0086 & 0.6352 & 0.6084 & 0.040^{*} \\ \text { C27C } & 0.93266(19) & 0.49686(4) & 0.32602(17) & 0.0332(5) \\ \text { H19C } & 0.8807 & 0.4997 & 0.3823 & 0.040^{*} \\ \text { N1A } & 0.27915(14) & 0.69645(3) & 1.07744(13) & 0.0248(4) \\ \text { N1B } & 0.68818(13) & 0.53510(3) & 0.43417(13) & 0.0230(4) \\ \text { N1C } & 0.69395(14) & 0.63399(3) & -0.06137(13) & 0.0248(4) \\ \text { N2A } & 0.16966(15) & 0.79942(3) & 0.81534(14) & 0.0268(4) \\ \text { N2B } & 0.80552(15) & 0.63521(4) & 0.70989(14) & 0.0263(4) \\ \text { N2C } & 0.82550(15) & 0.53481(3) & 0.21699(14) & 0.0254(4) \\ \text { H20A } & 0.2198(19) & 0.8020(5) & 0.7589(19) & 0.044(7)^{*} \\ \text { H20B } & 0.7628(19) & 0.6364(4) & 0.7690(18) & 0.038(6)^{*} \\ \text { H20C } & 0.7737(17) & 0.5329(4) & 0.2703(17) & 0.027(6)^{*} \\ & & & \end{array}$

Atomic displacement parameters $\left(\AA^{2}\right)$

\begin{tabular}{|c|c|c|c|c|c|c|}
\hline & $U^{11}$ & $U^{22}$ & $U^{33}$ & $U^{12}$ & $U^{13}$ & $U^{23}$ \\
\hline $\mathrm{C} 1 \mathrm{~A}$ & $0.0274(11)$ & $0.0251(11)$ & $0.0284(11)$ & 0.0057 (9) & $0.0130(9)$ & $0.0017(9)$ \\
\hline C1B & $0.0298(12)$ & $0.0254(11)$ & $0.0321(12)$ & $-0.0082(9)$ & $0.0153(10)$ & $0.0008(9)$ \\
\hline $\mathrm{C} 1 \mathrm{C}$ & $0.0297(12)$ & $0.0325(12)$ & $0.0349(12)$ & $0.0139(10)$ & $0.0156(10)$ & $0.0094(10)$ \\
\hline $\mathrm{C} 2 \mathrm{~A}$ & 0.0385 (13) & $0.0282(12)$ & $0.0285(12)$ & $0.0030(10)$ & $0.0092(10)$ & $0.0019(9)$ \\
\hline $\mathrm{C} 2 \mathrm{~B}$ & $0.0411(13)$ & 0.0237 (11) & $0.0346(13)$ & $-0.0042(10)$ & 0.0094 (11) & $0.0004(10)$ \\
\hline $\mathrm{C} 2 \mathrm{C}$ & $0.0297(12)$ & $0.0417(14)$ & $0.0367(13)$ & $0.0101(10)$ & $0.0078(10)$ & $0.0099(11)$ \\
\hline $\mathrm{C} 3 \mathrm{~A}$ & $0.0375(13)$ & $0.0336(13)$ & $0.0356(12)$ & $-0.0017(10)$ & $0.0092(10)$ & $-0.0045(10)$ \\
\hline $\mathrm{C} 3 \mathrm{~B}$ & $0.0458(14)$ & $0.0340(13)$ & $0.0391(13)$ & $-0.0011(11)$ & $0.0088(11)$ & $0.0055(11)$ \\
\hline $\mathrm{C} 3 \mathrm{C}$ & $0.0318(13)$ & 0.0597 (16) & $0.0409(14)$ & $0.0071(12)$ & $0.0027(11)$ & $0.0084(12)$ \\
\hline $\mathrm{C} 4 \mathrm{~A}$ & $0.0332(12)$ & $0.0330(13)$ & $0.0493(15)$ & $-0.0052(10)$ & $0.0112(12)$ & $-0.0053(11)$ \\
\hline C4B & $0.0422(14)$ & $0.0296(13)$ & $0.0554(16)$ & $0.0042(11)$ & $0.0137(13)$ & $0.0075(12)$ \\
\hline $\mathrm{C} 4 \mathrm{C}$ & 0.0355 (14) & $0.076(2)$ & $0.0398(14)$ & $0.0160(14)$ & $0.0055(11)$ & $0.0158(14)$ \\
\hline $\mathrm{C} 5 \mathrm{~A}$ & $0.0339(12)$ & $0.0231(12)$ & $0.0579(16)$ & $-0.0005(10)$ & $0.0161(12)$ & $0.0050(11)$ \\
\hline $\mathrm{C} 5 \mathrm{~B}$ & $0.0386(13)$ & $0.0252(12)$ & $0.0544(16)$ & $-0.0025(10)$ & $0.0162(12)$ & $-0.0051(11)$ \\
\hline $\mathrm{C} 5 \mathrm{C}$ & $0.0414(14)$ & $0.0595(17)$ & $0.0442(15)$ & $0.0261(13)$ & $0.0170(12)$ & $0.0282(13)$ \\
\hline C6A & $0.0268(11)$ & $0.0275(12)$ & $0.0409(13)$ & $0.0077(9)$ & $0.0147(10)$ & $0.0067(10)$ \\
\hline C6B & $0.0304(11)$ & $0.0252(12)$ & $0.0381(13)$ & $-0.0060(10)$ & $0.0159(10)$ & $-0.0042(10)$ \\
\hline $\mathrm{C} 6 \mathrm{C}$ & $0.0348(13)$ & $0.0364(13)$ & $0.0422(14)$ & $0.0162(11)$ & $0.0202(11)$ & $0.0156(11)$ \\
\hline C7A & $0.0334(12)$ & $0.0289(12)$ & $0.0459(14)$ & $0.0082(10)$ & $0.0174(11)$ & $0.0130(11)$ \\
\hline C7B & $0.0344(12)$ & $0.0258(12)$ & $0.0426(14)$ & $-0.0098(10)$ & $0.0189(11)$ & $-0.0139(10)$ \\
\hline $\mathrm{C} 7 \mathrm{C}$ & $0.0447(14)$ & $0.0351(14)$ & $0.0564(16)$ & $0.0174(11)$ & $0.0299(13)$ & $0.0252(12)$ \\
\hline $\mathrm{C} 8 \mathrm{~A}$ & $0.0259(11)$ & 0.0403 (13) & $0.0312(12)$ & $0.0144(10)$ & $0.0112(10)$ & $0.0121(10)$ \\
\hline $\mathrm{C} 8 \mathrm{~B}$ & $0.0273(11)$ & $0.0320(12)$ & $0.0330(12)$ & $-0.0104(10)$ & $0.0157(10)$ & $-0.0080(10)$ \\
\hline $\mathrm{C} 8 \mathrm{C}$ & $0.0429(14)$ & $0.0253(12)$ & $0.0550(16)$ & $0.0077(10)$ & $0.0289(12)$ & $0.0088(11)$ \\
\hline C9A & $0.0387(14)$ & $0.0470(15)$ & $0.0396(14)$ & $0.0156(12)$ & $0.0062(11)$ & $0.0124(12)$ \\
\hline C9B & $0.0378(13)$ & $0.0424(14)$ & $0.0358(13)$ & $-0.0136(11)$ & $0.0104(11)$ & $-0.0163(11)$ \\
\hline $\mathrm{C} 9 \mathrm{C}$ & $0.0625(18)$ & $0.0279(14)$ & $0.076(2)$ & $0.0044(12)$ & $0.0374(16)$ & $0.0078(13)$ \\
\hline C10A & $0.0365(14)$ & $0.0704(18)$ & $0.0319(13)$ & 0.0195 (13) & $-0.0019(11)$ & 0.0107 (13) \\
\hline $\mathrm{C} 10 \mathrm{~B}$ & $0.0377(14)$ & $0.0572(17)$ & $0.0332(13)$ & $-0.0104(12)$ & $-0.0005(11)$ & $-0.0129(12)$ \\
\hline $\mathrm{C} 10 \mathrm{C}$ & $0.0690(19)$ & $0.0333(15)$ & 0.075 (2) & $-0.0108(13)$ & $0.0412(17)$ & $-0.0121(14)$ \\
\hline C11A & $0.0284(12)$ & $0.0577(16)$ & $0.0413(14)$ & $0.0047(11)$ & $0.0002(11)$ & $-0.0017(12)$ \\
\hline C11B & $0.0360(13)$ & $0.0451(14)$ & $0.0399(14)$ & $-0.0018(11)$ & -0.0014 & -0.0065 \\
\hline
\end{tabular}




\begin{tabular}{|c|c|c|c|c|c|c|}
\hline $\mathrm{C} 11 \mathrm{C}$ & $0.0623(17)$ & $0.0406(15)$ & $0.0514(15)$ & $-0.0098(13)$ & $0.0243(13)$ & $-0.0112(13)$ \\
\hline C12A & $0.0272(12)$ & $0.0412(13)$ & $0.0334(12)$ & $0.0077(10)$ & $0.0035(10)$ & $0.0021(10)$ \\
\hline $\mathrm{C} 12 \mathrm{~B}$ & $0.0301(12)$ & $0.0309(12)$ & $0.0336(12)$ & $-0.0051(10)$ & $0.0059(10)$ & $-0.0052(10)$ \\
\hline $\mathrm{C} 12 \mathrm{C}$ & $0.0547(15)$ & 0.0297 (13) & $0.0417(14)$ & $-0.0007(11)$ & $0.0202(12)$ & $-0.0016(11)$ \\
\hline C13A & $0.0231(11)$ & $0.0324(12)$ & $0.0275(11)$ & $0.0084(9)$ & $0.0096(9)$ & $0.0021(9)$ \\
\hline C13B & $0.0259(11)$ & $0.0271(11)$ & $0.0290(11)$ & $-0.0100(9)$ & $0.0126(9)$ & $-0.0046(9)$ \\
\hline $\mathrm{C} 13 \mathrm{C}$ & $0.0407(13)$ & $0.0239(12)$ & $0.0384(13)$ & $0.0075(10)$ & $0.0233(11)$ & $0.0019(10)$ \\
\hline C14A & $0.0260(11)$ & $0.0261(11)$ & $0.0253(11)$ & $0.0069(9)$ & $0.0101(9)$ & $0.0038(9)$ \\
\hline C14B & $0.0263(11)$ & $0.0231(11)$ & $0.0275(11)$ & $-0.0068(9)$ & $0.0121(9)$ & $-0.0031(9)$ \\
\hline $\mathrm{C} 14 \mathrm{C}$ & $0.0347(12)$ & $0.0252(12)$ & $0.0326(12)$ & $0.0094(10)$ & $0.0170(10)$ & $0.0044(9)$ \\
\hline $\mathrm{C} 15 \mathrm{~A}$ & $0.0286(11)$ & $0.0272(11)$ & $0.0237(11)$ & $0.0017(9)$ & $0.0071(9)$ & $0.0014(9)$ \\
\hline C15B & $0.0280(11)$ & $0.0264(11)$ & $0.0212(10)$ & $-0.0021(9)$ & $0.0078(9)$ & $-0.0015(9)$ \\
\hline $\mathrm{C} 15 \mathrm{C}$ & $0.0351(12)$ & $0.0258(11)$ & $0.0230(11)$ & $0.0029(9)$ & $0.0094(9)$ & $0.0024(9)$ \\
\hline C16A & $0.0278(11)$ & $0.0212(10)$ & $0.0204(10)$ & $0.0001(9)$ & $0.0014(9)$ & $-0.0015(8)$ \\
\hline C16B & $0.0227(10)$ & $0.0203(10)$ & $0.0194(10)$ & $0.0003(8)$ & $-0.0013(8)$ & $0.0010(8)$ \\
\hline $\mathrm{C} 16 \mathrm{C}$ & $0.0282(11)$ & $0.0198(10)$ & $0.0165(10)$ & $0.0005(9)$ & $0.0020(9)$ & $-0.0005(8)$ \\
\hline C17A & $0.0242(11)$ & $0.0233(11)$ & $0.0260(11)$ & $0.0022(9)$ & $0.0038(9)$ & $-0.0020(9)$ \\
\hline C17B & $0.0214(10)$ & $0.0212(11)$ & $0.0251(11)$ & $-0.0020(8)$ & $0.0041(9)$ & $0.0037(9)$ \\
\hline $\mathrm{C} 17 \mathrm{C}$ & $0.0326(12)$ & $0.0253(11)$ & $0.0242(11)$ & $0.0017(9)$ & $0.0116(9)$ & $0.0044(9)$ \\
\hline C18A & $0.0269(11)$ & $0.0243(11)$ & $0.0216(10)$ & $-0.0027(9)$ & $0.0057(9)$ & $-0.0017(9)$ \\
\hline C18B & $0.0250(11)$ & $0.0233(11)$ & $0.0207(10)$ & $0.0022(9)$ & $0.0080(9)$ & $-0.0004(8)$ \\
\hline $\mathrm{C} 18 \mathrm{C}$ & $0.0248(11)$ & 0.0257 (11) & $0.0307(11)$ & $0.0045(9)$ & $0.0096(9)$ & $0.0027(9)$ \\
\hline C19A & $0.0252(11)$ & $0.0199(10)$ & $0.0200(10)$ & $-0.0020(9)$ & $-0.0006(8)$ & $-0.0027(8)$ \\
\hline C19B & $0.0233(11)$ & $0.0191(10)$ & $0.0202(10)$ & $0.0005(8)$ & $0.0011(8)$ & $0.0010(8)$ \\
\hline C19C & $0.0263(11)$ & $0.0191(10)$ & $0.0173(10)$ & $-0.0021(8)$ & $0.0001(8)$ & $-0.0016(8)$ \\
\hline C20A & $0.0327(12)$ & $0.0291(12)$ & $0.0265(11)$ & $0.0082(9)$ & $0.0095(10)$ & $-0.0006(9)$ \\
\hline C20B & $0.0328(12)$ & $0.0292(12)$ & $0.0256(11)$ & $-0.0125(10)$ & $0.0102(9)$ & $-0.0023(9)$ \\
\hline C20C & $0.0273(11)$ & $0.0222(10)$ & $0.0207(10)$ & $-0.0016(9)$ & $0.0075(9)$ & $-0.0011(8)$ \\
\hline $\mathrm{C} 21 \mathrm{~A}$ & $0.0359(12)$ & $0.0312(12)$ & $0.0205(11)$ & $0.0055(10)$ & $0.0093(9)$ & $0.0022(9)$ \\
\hline C21B & $0.0358(12)$ & $0.0300(12)$ & $0.0192(10)$ & $-0.0062(10)$ & $0.0097(9)$ & $-0.0027(9)$ \\
\hline $\mathrm{C} 21 \mathrm{C}$ & $0.0229(10)$ & $0.0247(11)$ & $0.0235(10)$ & $0.0041(9)$ & $0.0040(9)$ & $-0.0010(9)$ \\
\hline $\mathrm{C} 22 \mathrm{~A}$ & $0.0287(11)$ & $0.0238(11)$ & $0.0186(10)$ & $0.0020(9)$ & $0.0016(9)$ & $-0.0018(8)$ \\
\hline $\mathrm{C} 22 \mathrm{~B}$ & $0.0271(11)$ & $0.0214(11)$ & $0.0218(10)$ & $-0.0026(9)$ & $-0.0012(9)$ & $0.0021(9)$ \\
\hline $\mathrm{C} 22 \mathrm{C}$ & $0.0246(11)$ & $0.0207(10)$ & $0.0248(11)$ & $-0.0021(9)$ & $0.0009(9)$ & $-0.0019(9)$ \\
\hline $\mathrm{C} 23 \mathrm{~A}$ & $0.0405(14)$ & $0.0350(13)$ & $0.0420(14)$ & $0.0050(11)$ & $0.0122(11)$ & $0.0127(11)$ \\
\hline C23B & $0.0465(14)$ & $0.0348(13)$ & $0.0421(14)$ & $-0.0084(11)$ & $0.0092(11)$ & $-0.0112(11)$ \\
\hline $\mathrm{C} 23 \mathrm{C}$ & $0.0387(13)$ & $0.0277(12)$ & $0.0308(12)$ & $0.0060(10)$ & $0.0085(10)$ & $0.0028(10)$ \\
\hline C24A & $0.0505(15)$ & $0.0333(13)$ & $0.0451(14)$ & 0.0077 (12) & $0.0029(12)$ & $0.0119(11)$ \\
\hline C24B & $0.0685(18)$ & $0.0318(14)$ & $0.0498(16)$ & $-0.0171(13)$ & $-0.0022(14)$ & $-0.0102(12)$ \\
\hline $\mathrm{C} 24 \mathrm{C}$ & $0.0323(12)$ & $0.0340(13)$ & $0.0380(13)$ & $0.0055(10)$ & $0.0058(10)$ & $-0.0069(11)$ \\
\hline $\mathrm{C} 25 \mathrm{~A}$ & $0.0377(13)$ & $0.0317(13)$ & $0.0351(13)$ & $0.0123(10)$ & $-0.0074(11)$ & $-0.0046(10)$ \\
\hline $\mathrm{C} 25 \mathrm{~B}$ & $0.0501(16)$ & $0.0398(14)$ & $0.0436(14)$ & $-0.0243(12)$ & $-0.0147(13)$ & $0.0132(12)$ \\
\hline $\mathrm{C} 25 \mathrm{C}$ & $0.0334(13)$ & $0.0348(13)$ & $0.0416(14)$ & $0.0108(10)$ & $-0.0068(11)$ & $-0.0041(11)$ \\
\hline C26A & $0.0298(12)$ & $0.0488(15)$ & $0.0480(15)$ & $0.0075(11)$ & $0.0080(11)$ & $0.0059(12)$ \\
\hline C26B & $0.0314(13)$ & $0.0545(16)$ & $0.0411(14)$ & $-0.0125(12)$ & $-0.0004(11)$ & $0.0079(12)$ \\
\hline $\mathrm{C} 26 \mathrm{C}$ & $0.0433(14)$ & $0.0365(13)$ & $0.0390(13)$ & $0.0102(11)$ & $-0.0030(11)$ & $0.0096(11)$ \\
\hline $\mathrm{C} 27 \mathrm{~A}$ & $0.0333(12)$ & $0.0334(13)$ & $0.0423(13)$ & $0.0032(10)$ & $0.0071(11)$ & $0.0139(10)$ \\
\hline C27B & $0.0290(12)$ & $0.0363(13)$ & $0.0334(12)$ & $-0.0065(10)$ & $0.0014(10)$ & $-0.0030(10)$ \\
\hline
\end{tabular}




\begin{tabular}{lllllll}
\hline C27C & $0.0341(12)$ & $0.0347(12)$ & $0.0312(12)$ & $0.0025(10)$ & $0.0063(10)$ & $0.0052(10)$ \\
N1A & $0.0264(9)$ & $0.0244(9)$ & $0.0239(9)$ & $0.0050(7)$ & $0.0041(7)$ & $0.0008(8)$ \\
N1B & $0.0236(9)$ & $0.0229(9)$ & $0.0228(9)$ & $-0.0041(7)$ & $0.0042(7)$ & $-0.0015(7)$ \\
N1C & $0.0283(9)$ & $0.0237(9)$ & $0.0229(9)$ & $0.0037(8)$ & $0.0055(7)$ & $0.0018(7)$ \\
N2A & $0.0292(10)$ & $0.0254(10)$ & $0.0271(9)$ & $0.0047(8)$ & $0.0088(8)$ & $0.0032(8)$ \\
N2B & $0.0287(10)$ & $0.0262(10)$ & $0.0251(9)$ & $-0.0058(8)$ & $0.0085(8)$ & $-0.0062(8)$ \\
N2C & $0.0287(10)$ & $0.0248(9)$ & $0.0243(9)$ & $0.0044(8)$ & $0.0093(8)$ & $0.0051(8)$ \\
\hline
\end{tabular}

Geometric parameters $\left(\AA,{ }^{\circ}\right)$

\begin{tabular}{|c|c|c|c|}
\hline $\mathrm{C} 1 \mathrm{~A}-\mathrm{C} 14 \mathrm{~A}$ & $1.417(3)$ & $\mathrm{C} 15 \mathrm{~B}-\mathrm{N} 1 \mathrm{~B}$ & $1.276(2)$ \\
\hline $\mathrm{C} 1 \mathrm{~A}-\mathrm{C} 2 \mathrm{~A}$ & $1.426(3)$ & $\mathrm{C} 15 \mathrm{~B}-\mathrm{H} 10 \mathrm{~B}$ & 0.9300 \\
\hline $\mathrm{C} 1 \mathrm{~A}-\mathrm{C} 6 \mathrm{~A}$ & $1.432(3)$ & $\mathrm{C} 15 \mathrm{C}-\mathrm{N} 1 \mathrm{C}$ & $1.271(2)$ \\
\hline $\mathrm{C} 1 \mathrm{~B}-\mathrm{C} 14 \mathrm{~B}$ & $1.417(3)$ & $\mathrm{C} 15 \mathrm{C}-\mathrm{H} 10 \mathrm{C}$ & 0.9300 \\
\hline $\mathrm{C} 1 \mathrm{~B}-\mathrm{C} 2 \mathrm{~B}$ & $1.427(3)$ & $\mathrm{C} 16 \mathrm{~A}-\mathrm{C} 21 \mathrm{~A}$ & $1.378(3)$ \\
\hline $\mathrm{C} 1 \mathrm{~B}-\mathrm{C} 6 \mathrm{~B}$ & $1.433(3)$ & $\mathrm{C} 16 \mathrm{~A}-\mathrm{C} 17 \mathrm{~A}$ & $1.393(3)$ \\
\hline $\mathrm{C} 1 \mathrm{C}-\mathrm{C} 14 \mathrm{C}$ & $1.410(3)$ & $\mathrm{C} 16 \mathrm{~A}-\mathrm{N} 1 \mathrm{~A}$ & $1.422(2)$ \\
\hline $\mathrm{C} 1 \mathrm{C}-\mathrm{C} 2 \mathrm{C}$ & $1.423(3)$ & $\mathrm{C} 16 \mathrm{~B}-\mathrm{C} 21 \mathrm{~B}$ & $1.381(3)$ \\
\hline $\mathrm{C} 1 \mathrm{C}-\mathrm{C} 6 \mathrm{C}$ & $1.437(3)$ & $\mathrm{C} 16 \mathrm{~B}-\mathrm{C} 17 \mathrm{~B}$ & $1.391(2)$ \\
\hline $\mathrm{C} 2 \mathrm{~A}-\mathrm{C} 3 \mathrm{~A}$ & $1.355(3)$ & $\mathrm{C} 16 \mathrm{~B}-\mathrm{N} 1 \mathrm{~B}$ & $1.419(2)$ \\
\hline $\mathrm{C} 2 \mathrm{~A}-\mathrm{H} 1 \mathrm{~A}$ & 0.9300 & $\mathrm{C} 16 \mathrm{C}-\mathrm{C} 17 \mathrm{C}$ & $1.376(3)$ \\
\hline $\mathrm{C} 2 \mathrm{~B}-\mathrm{C} 3 \mathrm{~B}$ & $1.359(3)$ & $\mathrm{C} 16 \mathrm{C}-\mathrm{C} 21 \mathrm{C}$ & $1.393(3)$ \\
\hline $\mathrm{C} 2 \mathrm{~B}-\mathrm{H} 1 \mathrm{~B}$ & 0.9300 & $\mathrm{C} 16 \mathrm{C}-\mathrm{N} 1 \mathrm{C}$ & $1.420(2)$ \\
\hline $\mathrm{C} 2 \mathrm{C}-\mathrm{C} 3 \mathrm{C}$ & $1.352(3)$ & $\mathrm{C} 17 \mathrm{~A}-\mathrm{C} 18 \mathrm{~A}$ & $1.379(3)$ \\
\hline $\mathrm{C} 2 \mathrm{C}-\mathrm{H} 1 \mathrm{C}$ & 0.9300 & $\mathrm{C} 17 \mathrm{~A}-\mathrm{H} 11 \mathrm{~A}$ & 0.9300 \\
\hline $\mathrm{C} 3 \mathrm{~A}-\mathrm{C} 4 \mathrm{~A}$ & $1.413(3)$ & $\mathrm{C} 17 \mathrm{~B}-\mathrm{C} 18 \mathrm{~B}$ & $1.375(2)$ \\
\hline $\mathrm{C} 3 \mathrm{~A}-\mathrm{H} 2 \mathrm{~A}$ & 0.9300 & C17B-H11B & 0.9300 \\
\hline $\mathrm{C} 3 \mathrm{~B}-\mathrm{C} 4 \mathrm{~B}$ & $1.410(3)$ & $\mathrm{C} 17 \mathrm{C}-\mathrm{C} 18 \mathrm{C}$ & $1.384(3)$ \\
\hline $\mathrm{C} 3 \mathrm{~B}-\mathrm{H} 2 \mathrm{~B}$ & 0.9300 & $\mathrm{C} 17 \mathrm{C}-\mathrm{H} 11 \mathrm{C}$ & 0.9300 \\
\hline $\mathrm{C} 3 \mathrm{C}-\mathrm{C} 4 \mathrm{C}$ & $1.407(3)$ & $\mathrm{C} 18 \mathrm{~A}-\mathrm{C} 19 \mathrm{~A}$ & $1.396(3)$ \\
\hline $\mathrm{C} 3 \mathrm{C}-\mathrm{H} 2 \mathrm{C}$ & 0.9300 & $\mathrm{C} 18 \mathrm{~A}-\mathrm{H} 12 \mathrm{~A}$ & 0.9300 \\
\hline $\mathrm{C} 4 \mathrm{~A}-\mathrm{C} 5 \mathrm{~A}$ & $1.344(3)$ & $\mathrm{C} 18 \mathrm{~B}-\mathrm{C} 19 \mathrm{~B}$ & $1.397(3)$ \\
\hline $\mathrm{C} 4 \mathrm{~A}-\mathrm{H} 3 \mathrm{~A}$ & 0.9300 & $\mathrm{C} 18 \mathrm{~B}-\mathrm{H} 12 \mathrm{~B}$ & 0.9300 \\
\hline $\mathrm{C} 4 \mathrm{~B}-\mathrm{C} 5 \mathrm{~B}$ & $1.351(3)$ & $\mathrm{C} 18 \mathrm{C}-\mathrm{C} 19 \mathrm{C}$ & $1.394(3)$ \\
\hline $\mathrm{C} 4 \mathrm{~B}-\mathrm{H} 3 \mathrm{~B}$ & 0.9300 & $\mathrm{C} 18 \mathrm{C}-\mathrm{H} 12 \mathrm{C}$ & 0.9300 \\
\hline $\mathrm{C} 4 \mathrm{C}-\mathrm{C} 5 \mathrm{C}$ & $1.350(3)$ & $\mathrm{C} 19 \mathrm{~A}-\mathrm{N} 2 \mathrm{~A}$ & $1.390(2)$ \\
\hline $\mathrm{C} 4 \mathrm{C}-\mathrm{H} 3 \mathrm{C}$ & 0.9300 & $\mathrm{C} 19 \mathrm{~A}-\mathrm{C} 20 \mathrm{~A}$ & $1.396(3)$ \\
\hline $\mathrm{C} 5 \mathrm{~A}-\mathrm{C} 6 \mathrm{~A}$ & $1.429(3)$ & $\mathrm{C} 19 \mathrm{~B}-\mathrm{N} 2 \mathrm{~B}$ & $1.385(2)$ \\
\hline $\mathrm{C} 5 \mathrm{~A}-\mathrm{H} 4 \mathrm{~A}$ & 0.9300 & $\mathrm{C} 19 \mathrm{~B}-\mathrm{C} 20 \mathrm{~B}$ & $1.393(3)$ \\
\hline $\mathrm{C} 5 \mathrm{~B}-\mathrm{C} 6 \mathrm{~B}$ & $1.420(3)$ & $\mathrm{C} 19 \mathrm{C}-\mathrm{N} 2 \mathrm{C}$ & $1.392(2)$ \\
\hline $\mathrm{C} 5 \mathrm{~B}-\mathrm{H} 4 \mathrm{~B}$ & 0.9300 & $\mathrm{C} 19 \mathrm{C}-\mathrm{C} 20 \mathrm{C}$ & $1.397(3)$ \\
\hline $\mathrm{C} 5 \mathrm{C}-\mathrm{C} 6 \mathrm{C}$ & $1.419(3)$ & $\mathrm{C} 20 \mathrm{~A}-\mathrm{C} 21 \mathrm{~A}$ & $1.383(3)$ \\
\hline $\mathrm{C} 5 \mathrm{C}-\mathrm{H} 4 \mathrm{C}$ & 0.9300 & $\mathrm{C} 20 \mathrm{~A}-\mathrm{H} 13 \mathrm{~A}$ & 0.9300 \\
\hline $\mathrm{C} 6 \mathrm{~A}-\mathrm{C} 7 \mathrm{~A}$ & $1.381(3)$ & $\mathrm{C} 20 \mathrm{~B}-\mathrm{C} 21 \mathrm{~B}$ & $1.381(3)$ \\
\hline $\mathrm{C} 6 \mathrm{~B}-\mathrm{C} 7 \mathrm{~B}$ & $1.386(3)$ & $\mathrm{C} 20 \mathrm{~B}-\mathrm{H} 13 \mathrm{~B}$ & 0.9300 \\
\hline $\mathrm{C} 6 \mathrm{C}-\mathrm{C} 7 \mathrm{C}$ & $1.390(3)$ & $\mathrm{C} 20 \mathrm{C}-\mathrm{C} 21 \mathrm{C}$ & $1.376(2)$ \\
\hline $\mathrm{C} 7 \mathrm{~A}-\mathrm{C} 8 \mathrm{~A}$ & $1.388(3)$ & $\mathrm{C} 20 \mathrm{C}-\mathrm{H} 14 \mathrm{C}$ & 0.9300 \\
\hline $\mathrm{C} 7 \mathrm{~A}-\mathrm{H} 5 \mathrm{~A}$ & 0.9300 & $\mathrm{C} 21 \mathrm{~A}-\mathrm{H} 14 \mathrm{~A}$ & 0.9300 \\
\hline
\end{tabular}




\begin{tabular}{|c|c|c|c|}
\hline $\mathrm{C} 7 \mathrm{~B}-\mathrm{C} 8 \mathrm{~B}$ & $1.388(3)$ & $\mathrm{C} 21 \mathrm{~B}-\mathrm{H} 14 \mathrm{~B}$ & 0.9300 \\
\hline $\mathrm{C} 7 \mathrm{~B}-\mathrm{H} 5 \mathrm{~B}$ & 0.9300 & $\mathrm{C} 21 \mathrm{C}-\mathrm{H} 13 \mathrm{C}$ & 0.9300 \\
\hline $\mathrm{C} 7 \mathrm{C}-\mathrm{C} 8 \mathrm{C}$ & $1.385(3)$ & $\mathrm{C} 22 \mathrm{~A}-\mathrm{C} 27 \mathrm{~A}$ & $1.381(3)$ \\
\hline $\mathrm{C} 7 \mathrm{C}-\mathrm{H} 5 \mathrm{C}$ & 0.9300 & $\mathrm{C} 22 \mathrm{~A}-\mathrm{C} 23 \mathrm{~A}$ & $1.388(3)$ \\
\hline $\mathrm{C} 8 \mathrm{~A}-\mathrm{C} 9 \mathrm{~A}$ & $1.421(3)$ & $\mathrm{C} 22 \mathrm{~A}-\mathrm{N} 2 \mathrm{~A}$ & $1.396(2)$ \\
\hline $\mathrm{C} 8 \mathrm{~A}-\mathrm{C} 13 \mathrm{~A}$ & $1.437(3)$ & $\mathrm{C} 22 \mathrm{~B}-\mathrm{C} 27 \mathrm{~B}$ & $1.383(3)$ \\
\hline $\mathrm{C} 8 \mathrm{~B}-\mathrm{C} 9 \mathrm{~B}$ & $1.420(3)$ & $\mathrm{C} 22 \mathrm{~B}-\mathrm{C} 23 \mathrm{~B}$ & $1.390(3)$ \\
\hline $\mathrm{C} 8 \mathrm{~B}-\mathrm{C} 13 \mathrm{~B}$ & $1.437(3)$ & $\mathrm{C} 22 \mathrm{~B}-\mathrm{N} 2 \mathrm{~B}$ & $1.394(2)$ \\
\hline $\mathrm{C} 8 \mathrm{C}-\mathrm{C} 9 \mathrm{C}$ & $1.422(3)$ & $\mathrm{C} 22 \mathrm{C}-\mathrm{C} 23 \mathrm{C}$ & $1.388(3)$ \\
\hline $\mathrm{C} 8 \mathrm{C}-\mathrm{C} 13 \mathrm{C}$ & $1.436(3)$ & $\mathrm{C} 22 \mathrm{C}-\mathrm{N} 2 \mathrm{C}$ & $1.392(2)$ \\
\hline $\mathrm{C} 9 \mathrm{~A}-\mathrm{C} 10 \mathrm{~A}$ & $1.340(3)$ & $\mathrm{C} 22 \mathrm{C}-\mathrm{C} 27 \mathrm{C}$ & $1.400(3)$ \\
\hline C9A-H6A & 0.9300 & $\mathrm{C} 23 \mathrm{~A}-\mathrm{C} 24 \mathrm{~A}$ & $1.371(3)$ \\
\hline $\mathrm{C} 9 \mathrm{~B}-\mathrm{C} 10 \mathrm{~B}$ & $1.341(3)$ & $\mathrm{C} 23 \mathrm{~A}-\mathrm{H} 15 \mathrm{~A}$ & 0.9300 \\
\hline C9B-H6B & 0.9300 & $\mathrm{C} 23 \mathrm{~B}-\mathrm{C} 24 \mathrm{~B}$ & $1.374(3)$ \\
\hline $\mathrm{C} 9 \mathrm{C}-\mathrm{C} 10 \mathrm{C}$ & $1.349(4)$ & $\mathrm{C} 23 \mathrm{~B}-\mathrm{H} 15 \mathrm{~B}$ & 0.9300 \\
\hline $\mathrm{C} 9 \mathrm{C}-\mathrm{H} 6 \mathrm{C}$ & 0.9300 & $\mathrm{C} 23 \mathrm{C}-\mathrm{C} 24 \mathrm{C}$ & $1.385(3)$ \\
\hline $\mathrm{C} 10 \mathrm{~A}-\mathrm{C} 11 \mathrm{~A}$ & $1.414(3)$ & $\mathrm{C} 23 \mathrm{C}-\mathrm{H} 15 \mathrm{C}$ & 0.9300 \\
\hline C10A-H7A & 0.9300 & $\mathrm{C} 24 \mathrm{~A}-\mathrm{C} 25 \mathrm{~A}$ & $1.370(3)$ \\
\hline $\mathrm{C} 10 \mathrm{~B}-\mathrm{C} 11 \mathrm{~B}$ & $1.411(3)$ & $\mathrm{C} 24 \mathrm{~A}-\mathrm{H} 16 \mathrm{~A}$ & 0.9300 \\
\hline $\mathrm{C} 10 \mathrm{~B}-\mathrm{H} 7 \mathrm{~B}$ & 0.9300 & $\mathrm{C} 24 \mathrm{~B}-\mathrm{C} 25 \mathrm{~B}$ & $1.363(3)$ \\
\hline $\mathrm{C} 10 \mathrm{C}-\mathrm{C} 11 \mathrm{C}$ & $1.415(3)$ & $\mathrm{C} 24 \mathrm{~B}-\mathrm{H} 16 \mathrm{~B}$ & 0.9300 \\
\hline $\mathrm{C} 10 \mathrm{C}-\mathrm{H} 7 \mathrm{C}$ & 0.9300 & $\mathrm{C} 24 \mathrm{C}-\mathrm{C} 25 \mathrm{C}$ & $1.371(3)$ \\
\hline $\mathrm{C} 11 \mathrm{~A}-\mathrm{C} 12 \mathrm{~A}$ & $1.358(3)$ & $\mathrm{C} 24 \mathrm{C}-\mathrm{H} 16 \mathrm{C}$ & 0.9300 \\
\hline $\mathrm{C} 11 \mathrm{~A}-\mathrm{H} 8 \mathrm{~A}$ & 0.9300 & $\mathrm{C} 25 \mathrm{~A}-\mathrm{C} 26 \mathrm{~A}$ & $1.369(3)$ \\
\hline $\mathrm{C} 11 \mathrm{~B}-\mathrm{C} 12 \mathrm{~B}$ & $1.357(3)$ & $\mathrm{C} 25 \mathrm{~A}-\mathrm{H} 17 \mathrm{~A}$ & 0.9300 \\
\hline $\mathrm{C} 11 \mathrm{~B}-\mathrm{H} 8 \mathrm{~B}$ & 0.9300 & $\mathrm{C} 25 \mathrm{~B}-\mathrm{C} 26 \mathrm{~B}$ & $1.372(3)$ \\
\hline $\mathrm{C} 11 \mathrm{C}-\mathrm{C} 12 \mathrm{C}$ & $1.358(3)$ & $\mathrm{C} 25 \mathrm{~B}-\mathrm{H} 17 \mathrm{~B}$ & 0.9300 \\
\hline $\mathrm{C} 11 \mathrm{C}-\mathrm{H} 8 \mathrm{C}$ & 0.9300 & $\mathrm{C} 25 \mathrm{C}-\mathrm{C} 26 \mathrm{C}$ & $1.382(3)$ \\
\hline $\mathrm{C} 12 \mathrm{~A}-\mathrm{C} 13 \mathrm{~A}$ & $1.428(3)$ & $\mathrm{C} 25 \mathrm{C}-\mathrm{H} 17 \mathrm{C}$ & 0.9300 \\
\hline C12A-H9A & 0.9300 & $\mathrm{C} 26 \mathrm{~A}-\mathrm{C} 27 \mathrm{~A}$ & $1.384(3)$ \\
\hline $\mathrm{C} 12 \mathrm{~B}-\mathrm{C} 13 \mathrm{~B}$ & $1.423(3)$ & $\mathrm{C} 26 \mathrm{~A}-\mathrm{H} 18 \mathrm{~A}$ & 0.9300 \\
\hline $\mathrm{C} 12 \mathrm{~B}-\mathrm{H} 9 \mathrm{~B}$ & 0.9300 & $\mathrm{C} 26 \mathrm{~B}-\mathrm{C} 27 \mathrm{~B}$ & $1.382(3)$ \\
\hline $\mathrm{C} 12 \mathrm{C}-\mathrm{C} 13 \mathrm{C}$ & $1.424(3)$ & $\mathrm{C} 26 \mathrm{~B}-\mathrm{H} 18 \mathrm{~B}$ & 0.9300 \\
\hline $\mathrm{C} 12 \mathrm{C}-\mathrm{H} 9 \mathrm{C}$ & 0.9300 & $\mathrm{C} 26 \mathrm{C}-\mathrm{C} 27 \mathrm{C}$ & $1.375(3)$ \\
\hline $\mathrm{C} 13 \mathrm{~A}-\mathrm{C} 14 \mathrm{~A}$ & $1.407(3)$ & $\mathrm{C} 26 \mathrm{C}-\mathrm{H} 18 \mathrm{C}$ & 0.9300 \\
\hline $\mathrm{C} 13 \mathrm{~B}-\mathrm{C} 14 \mathrm{~B}$ & $1.414(3)$ & $\mathrm{C} 27 \mathrm{~A}-\mathrm{H} 19 \mathrm{~A}$ & 0.9300 \\
\hline $\mathrm{C} 13 \mathrm{C}-\mathrm{C} 14 \mathrm{C}$ & $1.412(3)$ & $\mathrm{C} 27 \mathrm{~B}-\mathrm{H} 19 \mathrm{~B}$ & 0.9300 \\
\hline $\mathrm{C} 14 \mathrm{~A}-\mathrm{C} 15 \mathrm{~A}$ & $1.474(3)$ & $\mathrm{C} 27 \mathrm{C}-\mathrm{H} 19 \mathrm{C}$ & 0.9300 \\
\hline $\mathrm{C} 14 \mathrm{~B}-\mathrm{C} 15 \mathrm{~B}$ & $1.472(3)$ & $\mathrm{N} 2 \mathrm{~A}-\mathrm{H} 20 \mathrm{~A}$ & $0.92(2)$ \\
\hline $\mathrm{C} 14 \mathrm{C}-\mathrm{C} 15 \mathrm{C}$ & $1.476(3)$ & $\mathrm{N} 2 \mathrm{~B}-\mathrm{H} 20 \mathrm{~B}$ & $0.88(2)$ \\
\hline $\mathrm{C} 15 \mathrm{~A}-\mathrm{N} 1 \mathrm{~A}$ & $1.277(2)$ & $\mathrm{N} 2 \mathrm{C}-\mathrm{H} 20 \mathrm{C}$ & $0.90(2)$ \\
\hline $\mathrm{C} 15 \mathrm{~A}-\mathrm{H} 10 \mathrm{~A}$ & 0.9300 & & \\
\hline $\mathrm{C} 14 \mathrm{~A}-\mathrm{C} 1 \mathrm{~A}-\mathrm{C} 2 \mathrm{~A}$ & $123.43(18)$ & $\mathrm{C} 14 \mathrm{~A}-\mathrm{C} 15 \mathrm{~A}-\mathrm{H} 10 \mathrm{~A}$ & 118.5 \\
\hline $\mathrm{C} 14 \mathrm{~A}-\mathrm{C} 1 \mathrm{~A}-\mathrm{C} 6 \mathrm{~A}$ & $119.28(18)$ & $\mathrm{N} 1 \mathrm{~B}-\mathrm{C} 15 \mathrm{~B}-\mathrm{C} 14 \mathrm{~B}$ & $121.41(17)$ \\
\hline $\mathrm{C} 2 \mathrm{~A}-\mathrm{C} 1 \mathrm{~A}-\mathrm{C} 6 \mathrm{~A}$ & $117.23(19)$ & $\mathrm{N} 1 \mathrm{~B}-\mathrm{C} 15 \mathrm{~B}-\mathrm{H} 10 \mathrm{~B}$ & 119.3 \\
\hline $\mathrm{C} 14 \mathrm{~B}-\mathrm{C} 1 \mathrm{~B}-\mathrm{C} 2 \mathrm{~B}$ & $123.36(18)$ & $\mathrm{C} 14 \mathrm{~B}-\mathrm{C} 15 \mathrm{~B}-\mathrm{H} 10 \mathrm{~B}$ & 119.3 \\
\hline $\mathrm{C} 14 \mathrm{~B}-\mathrm{C} 1 \mathrm{~B}-\mathrm{C} 6 \mathrm{~B}$ & $119.08(19)$ & $\mathrm{N} 1 \mathrm{C}-\mathrm{C} 15 \mathrm{C}-\mathrm{C} 14 \mathrm{C}$ & 121.23 \\
\hline
\end{tabular}




\begin{tabular}{|c|c|c|c|}
\hline $\mathrm{C} 2 \mathrm{~B}-\mathrm{C} 1 \mathrm{~B}-\mathrm{C} 6 \mathrm{~B}$ & $117.41(19)$ & $\mathrm{N} 1 \mathrm{C}-\mathrm{C} 15 \mathrm{C}-\mathrm{H} 10 \mathrm{C}$ & 119.4 \\
\hline $\mathrm{C} 14 \mathrm{C}-\mathrm{C} 1 \mathrm{C}-\mathrm{C} 2 \mathrm{C}$ & $123.70(19)$ & $\mathrm{C} 14 \mathrm{C}-\mathrm{C} 15 \mathrm{C}-\mathrm{H} 10 \mathrm{C}$ & 119.4 \\
\hline $\mathrm{C} 14 \mathrm{C}-\mathrm{C} 1 \mathrm{C}-\mathrm{C} 6 \mathrm{C}$ & $119.3(2)$ & $\mathrm{C} 21 \mathrm{~A}-\mathrm{C} 16 \mathrm{~A}-\mathrm{C} 17 \mathrm{~A}$ & $118.38(17)$ \\
\hline $\mathrm{C} 2 \mathrm{C}-\mathrm{C} 1 \mathrm{C}-\mathrm{C} 6 \mathrm{C}$ & $117.0(2)$ & $\mathrm{C} 21 \mathrm{~A}-\mathrm{C} 16 \mathrm{~A}-\mathrm{N} 1 \mathrm{~A}$ & $118.30(17)$ \\
\hline $\mathrm{C} 3 \mathrm{~A}-\mathrm{C} 2 \mathrm{~A}-\mathrm{C} 1 \mathrm{~A}$ & $121.3(2)$ & $\mathrm{C} 17 \mathrm{~A}-\mathrm{C} 16 \mathrm{~A}-\mathrm{N} 1 \mathrm{~A}$ & $123.33(17)$ \\
\hline $\mathrm{C} 3 \mathrm{~A}-\mathrm{C} 2 \mathrm{~A}-\mathrm{H} 1 \mathrm{~A}$ & 119.3 & $\mathrm{C} 21 \mathrm{~B}-\mathrm{C} 16 \mathrm{~B}-\mathrm{C} 17 \mathrm{~B}$ & 118.03 (17) \\
\hline $\mathrm{C} 1 \mathrm{~A}-\mathrm{C} 2 \mathrm{~A}-\mathrm{H} 1 \mathrm{~A}$ & 119.3 & $\mathrm{C} 21 \mathrm{~B}-\mathrm{C} 16 \mathrm{~B}-\mathrm{N} 1 \mathrm{~B}$ & $117.53(16)$ \\
\hline $\mathrm{C} 3 \mathrm{~B}-\mathrm{C} 2 \mathrm{~B}-\mathrm{C} 1 \mathrm{~B}$ & $121.3(2)$ & $\mathrm{C} 17 \mathrm{~B}-\mathrm{C} 16 \mathrm{~B}-\mathrm{N} 1 \mathrm{~B}$ & $124.43(17)$ \\
\hline $\mathrm{C} 3 \mathrm{~B}-\mathrm{C} 2 \mathrm{~B}-\mathrm{H} 1 \mathrm{~B}$ & 119.3 & $\mathrm{C} 17 \mathrm{C}-\mathrm{C} 16 \mathrm{C}-\mathrm{C} 21 \mathrm{C}$ & $118.39(17)$ \\
\hline $\mathrm{C} 1 \mathrm{~B}-\mathrm{C} 2 \mathrm{~B}-\mathrm{H} 1 \mathrm{~B}$ & 119.3 & $\mathrm{C} 17 \mathrm{C}-\mathrm{C} 16 \mathrm{C}-\mathrm{N} 1 \mathrm{C}$ & $118.04(16)$ \\
\hline $\mathrm{C} 3 \mathrm{C}-\mathrm{C} 2 \mathrm{C}-\mathrm{C} 1 \mathrm{C}$ & $121.4(2)$ & $\mathrm{C} 21 \mathrm{C}-\mathrm{C} 16 \mathrm{C}-\mathrm{N} 1 \mathrm{C}$ & $123.58(17)$ \\
\hline $\mathrm{C} 3 \mathrm{C}-\mathrm{C} 2 \mathrm{C}-\mathrm{H} 1 \mathrm{C}$ & 119.3 & $\mathrm{C} 18 \mathrm{~A}-\mathrm{C} 17 \mathrm{~A}-\mathrm{C} 16 \mathrm{~A}$ & $120.59(18)$ \\
\hline $\mathrm{C} 1 \mathrm{C}-\mathrm{C} 2 \mathrm{C}-\mathrm{H} 1 \mathrm{C}$ & 119.3 & $\mathrm{C} 18 \mathrm{~A}-\mathrm{C} 17 \mathrm{~A}-\mathrm{H} 11 \mathrm{~A}$ & 119.7 \\
\hline $\mathrm{C} 2 \mathrm{~A}-\mathrm{C} 3 \mathrm{~A}-\mathrm{C} 4 \mathrm{~A}$ & $121.2(2)$ & $\mathrm{C} 16 \mathrm{~A}-\mathrm{C} 17 \mathrm{~A}-\mathrm{H} 11 \mathrm{~A}$ & 119.7 \\
\hline $\mathrm{C} 2 \mathrm{~A}-\mathrm{C} 3 \mathrm{~A}-\mathrm{H} 2 \mathrm{~A}$ & 119.4 & $\mathrm{C} 18 \mathrm{~B}-\mathrm{C} 17 \mathrm{~B}-\mathrm{C} 16 \mathrm{~B}$ & $120.63(17)$ \\
\hline $\mathrm{C} 4 \mathrm{~A}-\mathrm{C} 3 \mathrm{~A}-\mathrm{H} 2 \mathrm{~A}$ & 119.4 & $\mathrm{C} 18 \mathrm{~B}-\mathrm{C} 17 \mathrm{~B}-\mathrm{H} 11 \mathrm{~B}$ & 119.7 \\
\hline $\mathrm{C} 2 \mathrm{~B}-\mathrm{C} 3 \mathrm{~B}-\mathrm{C} 4 \mathrm{~B}$ & $120.9(2)$ & $\mathrm{C} 16 \mathrm{~B}-\mathrm{C} 17 \mathrm{~B}-\mathrm{H} 11 \mathrm{~B}$ & 119.7 \\
\hline $\mathrm{C} 2 \mathrm{~B}-\mathrm{C} 3 \mathrm{~B}-\mathrm{H} 2 \mathrm{~B}$ & 119.6 & $\mathrm{C} 16 \mathrm{C}-\mathrm{C} 17 \mathrm{C}-\mathrm{C} 18 \mathrm{C}$ & $121.43(18)$ \\
\hline $\mathrm{C} 4 \mathrm{~B}-\mathrm{C} 3 \mathrm{~B}-\mathrm{H} 2 \mathrm{~B}$ & 119.6 & $\mathrm{C} 16 \mathrm{C}-\mathrm{C} 17 \mathrm{C}-\mathrm{H} 11 \mathrm{C}$ & 119.3 \\
\hline $\mathrm{C} 2 \mathrm{C}-\mathrm{C} 3 \mathrm{C}-\mathrm{C} 4 \mathrm{C}$ & $121.4(2)$ & $\mathrm{C} 18 \mathrm{C}-\mathrm{C} 17 \mathrm{C}-\mathrm{H} 11 \mathrm{C}$ & 119.3 \\
\hline $\mathrm{C} 2 \mathrm{C}-\mathrm{C} 3 \mathrm{C}-\mathrm{H} 2 \mathrm{C}$ & 119.3 & $\mathrm{C} 17 \mathrm{~A}-\mathrm{C} 18 \mathrm{~A}-\mathrm{C} 19 \mathrm{~A}$ & $121.15(18)$ \\
\hline $\mathrm{C} 4 \mathrm{C}-\mathrm{C} 3 \mathrm{C}-\mathrm{H} 2 \mathrm{C}$ & 119.3 & $\mathrm{C} 17 \mathrm{~A}-\mathrm{C} 18 \mathrm{~A}-\mathrm{H} 12 \mathrm{~A}$ & 119.4 \\
\hline $\mathrm{C} 5 \mathrm{~A}-\mathrm{C} 4 \mathrm{~A}-\mathrm{C} 3 \mathrm{~A}$ & $119.5(2)$ & $\mathrm{C} 19 \mathrm{~A}-\mathrm{C} 18 \mathrm{~A}-\mathrm{H} 12 \mathrm{~A}$ & 119.4 \\
\hline $\mathrm{C} 5 \mathrm{~A}-\mathrm{C} 4 \mathrm{~A}-\mathrm{H} 3 \mathrm{~A}$ & 120.2 & $\mathrm{C} 17 \mathrm{~B}-\mathrm{C} 18 \mathrm{~B}-\mathrm{C} 19 \mathrm{~B}$ & $121.42(17)$ \\
\hline $\mathrm{C} 3 \mathrm{~A}-\mathrm{C} 4 \mathrm{~A}-\mathrm{H} 3 \mathrm{~A}$ & 120.2 & $\mathrm{C} 17 \mathrm{~B}-\mathrm{C} 18 \mathrm{~B}-\mathrm{H} 12 \mathrm{~B}$ & 119.3 \\
\hline $\mathrm{C} 5 \mathrm{~B}-\mathrm{C} 4 \mathrm{~B}-\mathrm{C} 3 \mathrm{~B}$ & $119.8(2)$ & $\mathrm{C} 19 \mathrm{~B}-\mathrm{C} 18 \mathrm{~B}-\mathrm{H} 12 \mathrm{~B}$ & 119.3 \\
\hline $\mathrm{C} 5 \mathrm{~B}-\mathrm{C} 4 \mathrm{~B}-\mathrm{H} 3 \mathrm{~B}$ & 120.1 & $\mathrm{C} 17 \mathrm{C}-\mathrm{C} 18 \mathrm{C}-\mathrm{C} 19 \mathrm{C}$ & $120.67(18)$ \\
\hline $\mathrm{C} 3 \mathrm{~B}-\mathrm{C} 4 \mathrm{~B}-\mathrm{H} 3 \mathrm{~B}$ & 120.1 & $\mathrm{C} 17 \mathrm{C}-\mathrm{C} 18 \mathrm{C}-\mathrm{H} 12 \mathrm{C}$ & 119.7 \\
\hline $\mathrm{C} 5 \mathrm{C}-\mathrm{C} 4 \mathrm{C}-\mathrm{C} 3 \mathrm{C}$ & $119.5(2)$ & $\mathrm{C} 19 \mathrm{C}-\mathrm{C} 18 \mathrm{C}-\mathrm{H} 12 \mathrm{C}$ & 119.7 \\
\hline $\mathrm{C} 5 \mathrm{C}-\mathrm{C} 4 \mathrm{C}-\mathrm{H} 3 \mathrm{C}$ & 120.3 & $\mathrm{~N} 2 \mathrm{~A}-\mathrm{C} 19 \mathrm{~A}-\mathrm{C} 18 \mathrm{~A}$ & $117.58(17)$ \\
\hline $\mathrm{C} 3 \mathrm{C}-\mathrm{C} 4 \mathrm{C}-\mathrm{H} 3 \mathrm{C}$ & 120.3 & $\mathrm{~N} 2 \mathrm{~A}-\mathrm{C} 19 \mathrm{~A}-\mathrm{C} 20 \mathrm{~A}$ & $124.68(18)$ \\
\hline $\mathrm{C} 4 \mathrm{~A}-\mathrm{C} 5 \mathrm{~A}-\mathrm{C} 6 \mathrm{~A}$ & $121.6(2)$ & $\mathrm{C} 18 \mathrm{~A}-\mathrm{C} 19 \mathrm{~A}-\mathrm{C} 20 \mathrm{~A}$ & $117.64(17)$ \\
\hline $\mathrm{C} 4 \mathrm{~A}-\mathrm{C} 5 \mathrm{~A}-\mathrm{H} 4 \mathrm{~A}$ & 119.2 & $\mathrm{~N} 2 \mathrm{~B}-\mathrm{C} 19 \mathrm{~B}-\mathrm{C} 20 \mathrm{~B}$ & $124.79(17)$ \\
\hline $\mathrm{C} 6 \mathrm{~A}-\mathrm{C} 5 \mathrm{~A}-\mathrm{H} 4 \mathrm{~A}$ & 119.2 & $\mathrm{~N} 2 \mathrm{~B}-\mathrm{C} 19 \mathrm{~B}-\mathrm{C} 18 \mathrm{~B}$ & $117.73(17)$ \\
\hline $\mathrm{C} 4 \mathrm{~B}-\mathrm{C} 5 \mathrm{~B}-\mathrm{C} 6 \mathrm{~B}$ & $121.6(2)$ & $\mathrm{C} 20 \mathrm{~B}-\mathrm{C} 19 \mathrm{~B}-\mathrm{C} 18 \mathrm{~B}$ & $117.38(17)$ \\
\hline $\mathrm{C} 4 \mathrm{~B}-\mathrm{C} 5 \mathrm{~B}-\mathrm{H} 4 \mathrm{~B}$ & 119.2 & $\mathrm{~N} 2 \mathrm{C}-\mathrm{C} 19 \mathrm{C}-\mathrm{C} 18 \mathrm{C}$ & $125.75(18)$ \\
\hline $\mathrm{C} 6 \mathrm{~B}-\mathrm{C} 5 \mathrm{~B}-\mathrm{H} 4 \mathrm{~B}$ & 119.2 & $\mathrm{~N} 2 \mathrm{C}-\mathrm{C} 19 \mathrm{C}-\mathrm{C} 20 \mathrm{C}$ & $116.84(17)$ \\
\hline $\mathrm{C} 4 \mathrm{C}-\mathrm{C} 5 \mathrm{C}-\mathrm{C} 6 \mathrm{C}$ & $121.4(2)$ & $\mathrm{C} 18 \mathrm{C}-\mathrm{C} 19 \mathrm{C}-\mathrm{C} 20 \mathrm{C}$ & $117.39(17)$ \\
\hline $\mathrm{C} 4 \mathrm{C}-\mathrm{C} 5 \mathrm{C}-\mathrm{H} 4 \mathrm{C}$ & 119.3 & $\mathrm{C} 21 \mathrm{~A}-\mathrm{C} 20 \mathrm{~A}-\mathrm{C} 19 \mathrm{~A}$ & $120.66(18)$ \\
\hline $\mathrm{C} 6 \mathrm{C}-\mathrm{C} 5 \mathrm{C}-\mathrm{H} 4 \mathrm{C}$ & 119.3 & $\mathrm{C} 21 \mathrm{~A}-\mathrm{C} 20 \mathrm{~A}-\mathrm{H} 13 \mathrm{~A}$ & 119.7 \\
\hline $\mathrm{C} 7 \mathrm{~A}-\mathrm{C} 6 \mathrm{~A}-\mathrm{C} 5 \mathrm{~A}$ & $121.79(19)$ & $\mathrm{C} 19 \mathrm{~A}-\mathrm{C} 20 \mathrm{~A}-\mathrm{H} 13 \mathrm{~A}$ & 119.7 \\
\hline $\mathrm{C} 7 \mathrm{~A}-\mathrm{C} 6 \mathrm{~A}-\mathrm{C} 1 \mathrm{~A}$ & $119.2(2)$ & $\mathrm{C} 21 \mathrm{~B}-\mathrm{C} 20 \mathrm{~B}-\mathrm{C} 19 \mathrm{~B}$ & $120.69(18)$ \\
\hline $\mathrm{C} 5 \mathrm{~A}-\mathrm{C} 6 \mathrm{~A}-\mathrm{C} 1 \mathrm{~A}$ & $119.0(2)$ & $\mathrm{C} 21 \mathrm{~B}-\mathrm{C} 20 \mathrm{~B}-\mathrm{H} 13 \mathrm{~B}$ & 119.7 \\
\hline $\mathrm{C} 7 \mathrm{~B}-\mathrm{C} 6 \mathrm{~B}-\mathrm{C} 5 \mathrm{~B}$ & $121.58(19)$ & $\mathrm{C} 19 \mathrm{~B}-\mathrm{C} 20 \mathrm{~B}-\mathrm{H} 13 \mathrm{~B}$ & 119.7 \\
\hline $\mathrm{C} 7 \mathrm{~B}-\mathrm{C} 6 \mathrm{~B}-\mathrm{C} 1 \mathrm{~B}$ & $119.42(19)$ & $\mathrm{C} 21 \mathrm{C}-\mathrm{C} 20 \mathrm{C}-\mathrm{C} 19 \mathrm{C}$ & $121.62(17)$ \\
\hline $\mathrm{C} 5 \mathrm{~B}-\mathrm{C} 6 \mathrm{~B}-\mathrm{C} 1 \mathrm{~B}$ & $119.0(2)$ & $\mathrm{C} 21 \mathrm{C}-\mathrm{C} 20 \mathrm{C}-\mathrm{H} 14 \mathrm{C}$ & 119.2 \\
\hline $\mathrm{C} 7 \mathrm{C}-\mathrm{C} 6 \mathrm{C}-\mathrm{C} 5 \mathrm{C}$ & $122.0(2)$ & $\mathrm{C} 19 \mathrm{C}-\mathrm{C} 20 \mathrm{C}-\mathrm{H} 14 \mathrm{C}$ & 119.2 \\
\hline $\mathrm{C} 7 \mathrm{C}-\mathrm{C} 6 \mathrm{C}-\mathrm{C} 1 \mathrm{C}$ & $118.7(2)$ & $\mathrm{C} 16 \mathrm{~A}-\mathrm{C} 21 \mathrm{~A}-\mathrm{C} 20 \mathrm{~A}$ & $121.21(18)$ \\
\hline
\end{tabular}




\begin{tabular}{|c|c|}
\hline $\mathrm{C} 5 \mathrm{C}-\mathrm{C} 6 \mathrm{C}-\mathrm{C} 1 \mathrm{C}$ & $119.2(2)$ \\
\hline $\mathrm{C} 6 \mathrm{~A}-\mathrm{C} 7 \mathrm{~A}-\mathrm{C} 8 \mathrm{~A}$ & $122.54(19)$ \\
\hline $\mathrm{C} 6 \mathrm{~A}-\mathrm{C} 7 \mathrm{~A}-\mathrm{H} 5 \mathrm{~A}$ & 118.7 \\
\hline $\mathrm{C} 8 \mathrm{~A}-\mathrm{C} 7 \mathrm{~A}-\mathrm{H} 5 \mathrm{~A}$ & 118.7 \\
\hline $\mathrm{C} 6 \mathrm{~B}-\mathrm{C} 7 \mathrm{~B}-\mathrm{C} 8 \mathrm{~B}$ & $122.51(19)$ \\
\hline $\mathrm{C} 6 \mathrm{~B}-\mathrm{C} 7 \mathrm{~B}-\mathrm{H} 5 \mathrm{~B}$ & 118.7 \\
\hline $\mathrm{C} 8 \mathrm{~B}-\mathrm{C} 7 \mathrm{~B}-\mathrm{H} 5 \mathrm{~B}$ & 118.7 \\
\hline $\mathrm{C} 8 \mathrm{C}-\mathrm{C} 7 \mathrm{C}-\mathrm{C} 6 \mathrm{C}$ & $122.8(2)$ \\
\hline $\mathrm{C} 8 \mathrm{C}-\mathrm{C} 7 \mathrm{C}-\mathrm{H} 5 \mathrm{C}$ & 118.6 \\
\hline $\mathrm{C} 6 \mathrm{C}-\mathrm{C} 7 \mathrm{C}-\mathrm{H} 5 \mathrm{C}$ & 118.6 \\
\hline $\mathrm{C} 7 \mathrm{~A}-\mathrm{C} 8 \mathrm{~A}-\mathrm{C} 9 \mathrm{~A}$ & $121.5(2)$ \\
\hline $\mathrm{C} 7 \mathrm{~A}-\mathrm{C} 8 \mathrm{~A}-\mathrm{C} 13 \mathrm{~A}$ & $119.27(19)$ \\
\hline $\mathrm{C} 9 \mathrm{~A}-\mathrm{C} 8 \mathrm{~A}-\mathrm{C} 13 \mathrm{~A}$ & $119.2(2)$ \\
\hline $\mathrm{C} 7 \mathrm{~B}-\mathrm{C} 8 \mathrm{~B}-\mathrm{C} 9 \mathrm{~B}$ & $121.69(19)$ \\
\hline $\mathrm{C} 7 \mathrm{~B}-\mathrm{C} 8 \mathrm{~B}-\mathrm{C} 13 \mathrm{~B}$ & $119.10(19)$ \\
\hline $\mathrm{C} 9 \mathrm{~B}-\mathrm{C} 8 \mathrm{~B}-\mathrm{C} 13 \mathrm{~B}$ & $119.2(2)$ \\
\hline $\mathrm{C} 7 \mathrm{C}-\mathrm{C} 8 \mathrm{C}-\mathrm{C} 9 \mathrm{C}$ & $122.3(2)$ \\
\hline $\mathrm{C} 7 \mathrm{C}-\mathrm{C} 8 \mathrm{C}-\mathrm{C} 13 \mathrm{C}$ & $119.4(2)$ \\
\hline $\mathrm{C} 9 \mathrm{C}-\mathrm{C} 8 \mathrm{C}-\mathrm{C} 13 \mathrm{C}$ & $118.3(2)$ \\
\hline $\mathrm{C} 10 \mathrm{~A}-\mathrm{C} 9 \mathrm{~A}-\mathrm{C} 8 \mathrm{~A}$ & $121.4(2)$ \\
\hline $\mathrm{C} 10 \mathrm{~A}-\mathrm{C} 9 \mathrm{~A}-\mathrm{H} 6 \mathrm{~A}$ & 119.3 \\
\hline $\mathrm{C} 8 \mathrm{~A}-\mathrm{C} 9 \mathrm{~A}-\mathrm{H} 6 \mathrm{~A}$ & 119.3 \\
\hline $\mathrm{C} 10 \mathrm{~B}-\mathrm{C} 9 \mathrm{~B}-\mathrm{C} 8 \mathrm{~B}$ & $121.5(2)$ \\
\hline $\mathrm{C} 10 \mathrm{~B}-\mathrm{C} 9 \mathrm{~B}-\mathrm{H} 6 \mathrm{~B}$ & 119.3 \\
\hline $\mathrm{C} 8 \mathrm{~B}-\mathrm{C} 9 \mathrm{~B}-\mathrm{H} 6 \mathrm{~B}$ & 119.3 \\
\hline $\mathrm{C} 10 \mathrm{C}-\mathrm{C} 9 \mathrm{C}-\mathrm{C} 8 \mathrm{C}$ & $122.3(2)$ \\
\hline $\mathrm{C} 10 \mathrm{C}-\mathrm{C} 9 \mathrm{C}-\mathrm{H} 6 \mathrm{C}$ & 118.9 \\
\hline $\mathrm{C} 8 \mathrm{C}-\mathrm{C} 9 \mathrm{C}-\mathrm{H} 6 \mathrm{C}$ & 118.9 \\
\hline $\mathrm{C} 9 \mathrm{~A}-\mathrm{C} 10 \mathrm{~A}-\mathrm{C} 11 \mathrm{~A}$ & $120.1(2)$ \\
\hline $\mathrm{C} 9 \mathrm{~A}-\mathrm{C} 10 \mathrm{~A}-\mathrm{H} 7 \mathrm{~A}$ & 120.0 \\
\hline $\mathrm{C} 11 \mathrm{~A}-\mathrm{C} 10 \mathrm{~A}-\mathrm{H} 7 \mathrm{~A}$ & 120.0 \\
\hline $\mathrm{C} 9 \mathrm{~B}-\mathrm{C} 10 \mathrm{~B}-\mathrm{C} 11 \mathrm{~B}$ & $119.7(2)$ \\
\hline $\mathrm{C} 9 \mathrm{~B}-\mathrm{C} 10 \mathrm{~B}-\mathrm{H} 7 \mathrm{~B}$ & 120.1 \\
\hline $\mathrm{C} 11 \mathrm{~B}-\mathrm{C} 10 \mathrm{~B}-\mathrm{H} 7 \mathrm{~B}$ & 120.1 \\
\hline $\mathrm{C} 9 \mathrm{C}-\mathrm{C} 10 \mathrm{C}-\mathrm{C} 11 \mathrm{C}$ & $119.4(2)$ \\
\hline $\mathrm{C} 9 \mathrm{C}-\mathrm{C} 10 \mathrm{C}-\mathrm{H} 7 \mathrm{C}$ & 120.3 \\
\hline $\mathrm{C} 11 \mathrm{C}-\mathrm{C} 10 \mathrm{C}-\mathrm{H} 7 \mathrm{C}$ & 120.3 \\
\hline $\mathrm{C} 12 \mathrm{~A}-\mathrm{C} 11 \mathrm{~A}-\mathrm{C} 10 \mathrm{~A}$ & $121.0(2)$ \\
\hline $\mathrm{C} 12 \mathrm{~A}-\mathrm{C} 11 \mathrm{~A}-\mathrm{H} 8 \mathrm{~A}$ & 119.5 \\
\hline $\mathrm{C} 10 \mathrm{~A}-\mathrm{C} 11 \mathrm{~A}-\mathrm{H} 8 \mathrm{~A}$ & 119.5 \\
\hline $\mathrm{C} 12 \mathrm{~B}-\mathrm{C} 11 \mathrm{~B}-\mathrm{C} 10 \mathrm{~B}$ & $121.4(2)$ \\
\hline $\mathrm{C} 12 \mathrm{~B}-\mathrm{C} 11 \mathrm{~B}-\mathrm{H} 8 \mathrm{~B}$ & 119.3 \\
\hline $\mathrm{C} 10 \mathrm{~B}-\mathrm{C} 11 \mathrm{~B}-\mathrm{H} 8 \mathrm{~B}$ & 119.3 \\
\hline $\mathrm{C} 12 \mathrm{C}-\mathrm{C} 11 \mathrm{C}-\mathrm{C} 10 \mathrm{C}$ & $120.8(3)$ \\
\hline $\mathrm{C} 12 \mathrm{C}-\mathrm{C} 11 \mathrm{C}-\mathrm{H} 8 \mathrm{C}$ & 119.6 \\
\hline $\mathrm{C} 10 \mathrm{C}-\mathrm{C} 11 \mathrm{C}-\mathrm{H} 8 \mathrm{C}$ & 119.6 \\
\hline $\mathrm{C} 11 \mathrm{~A}-\mathrm{C} 12 \mathrm{~A}-\mathrm{C} 13 \mathrm{~A}$ & $120.9(2)$ \\
\hline $\mathrm{C} 11 \mathrm{~A}-\mathrm{C} 12 \mathrm{~A}-\mathrm{H} 9 \mathrm{~A}$ & 119.5 \\
\hline
\end{tabular}

\begin{tabular}{|c|c|}
\hline $\mathrm{C} 16 \mathrm{~A}-\mathrm{C} 21 \mathrm{~A}-\mathrm{H} 14 \mathrm{~A}$ & 119.4 \\
\hline $\mathrm{C} 20 \mathrm{~A}-\mathrm{C} 21 \mathrm{~A}-\mathrm{H} 14 \mathrm{~A}$ & 119.4 \\
\hline $\mathrm{C} 20 \mathrm{~B}-\mathrm{C} 21 \mathrm{~B}-\mathrm{C} 16 \mathrm{~B}$ & $121.40(18)$ \\
\hline $\mathrm{C} 20 \mathrm{~B}-\mathrm{C} 21 \mathrm{~B}-\mathrm{H} 14 \mathrm{~B}$ & 119.3 \\
\hline $\mathrm{C} 16 \mathrm{~B}-\mathrm{C} 21 \mathrm{~B}-\mathrm{H} 14 \mathrm{~B}$ & 119.3 \\
\hline $\mathrm{C} 20 \mathrm{C}-\mathrm{C} 21 \mathrm{C}-\mathrm{C} 16 \mathrm{C}$ & $120.38(18)$ \\
\hline $\mathrm{C} 20 \mathrm{C}-\mathrm{C} 21 \mathrm{C}-\mathrm{H} 13 \mathrm{C}$ & 119.8 \\
\hline $\mathrm{C} 16 \mathrm{C}-\mathrm{C} 21 \mathrm{C}-\mathrm{H} 13 \mathrm{C}$ & 119.8 \\
\hline $\mathrm{C} 27 \mathrm{~A}-\mathrm{C} 22 \mathrm{~A}-\mathrm{C} 23 \mathrm{~A}$ & $117.34(18)$ \\
\hline $\mathrm{C} 27 \mathrm{~A}-\mathrm{C} 22 \mathrm{~A}-\mathrm{N} 2 \mathrm{~A}$ & $125.77(18)$ \\
\hline $\mathrm{C} 23 \mathrm{~A}-\mathrm{C} 22 \mathrm{~A}-\mathrm{N} 2 \mathrm{~A}$ & $116.86(18)$ \\
\hline $\mathrm{C} 27 \mathrm{~B}-\mathrm{C} 22 \mathrm{~B}-\mathrm{C} 23 \mathrm{~B}$ & $117.57(19)$ \\
\hline $\mathrm{C} 27 \mathrm{~B}-\mathrm{C} 22 \mathrm{~B}-\mathrm{N} 2 \mathrm{~B}$ & $125.31(18)$ \\
\hline $\mathrm{C} 23 \mathrm{~B}-\mathrm{C} 22 \mathrm{~B}-\mathrm{N} 2 \mathrm{~B}$ & $117.07(18)$ \\
\hline $\mathrm{C} 23 \mathrm{C}-\mathrm{C} 22 \mathrm{C}-\mathrm{N} 2 \mathrm{C}$ & $126.42(18)$ \\
\hline $\mathrm{C} 23 \mathrm{C}-\mathrm{C} 22 \mathrm{C}-\mathrm{C} 27 \mathrm{C}$ & $117.34(18)$ \\
\hline $\mathrm{N} 2 \mathrm{C}-\mathrm{C} 22 \mathrm{C}-\mathrm{C} 27 \mathrm{C}$ & $116.23(17)$ \\
\hline $\mathrm{C} 24 \mathrm{~A}-\mathrm{C} 23 \mathrm{~A}-\mathrm{C} 22 \mathrm{~A}$ & $121.8(2)$ \\
\hline $\mathrm{C} 24 \mathrm{~A}-\mathrm{C} 23 \mathrm{~A}-\mathrm{H} 15 \mathrm{~A}$ & 119.1 \\
\hline $\mathrm{C} 22 \mathrm{~A}-\mathrm{C} 23 \mathrm{~A}-\mathrm{H} 15 \mathrm{~A}$ & 119.1 \\
\hline $\mathrm{C} 24 \mathrm{~B}-\mathrm{C} 23 \mathrm{~B}-\mathrm{C} 22 \mathrm{~B}$ & $121.2(2)$ \\
\hline $\mathrm{C} 24 \mathrm{~B}-\mathrm{C} 23 \mathrm{~B}-\mathrm{H} 15 \mathrm{~B}$ & 119.4 \\
\hline $\mathrm{C} 22 \mathrm{~B}-\mathrm{C} 23 \mathrm{~B}-\mathrm{H} 15 \mathrm{~B}$ & 119.4 \\
\hline $\mathrm{C} 24 \mathrm{C}-\mathrm{C} 23 \mathrm{C}-\mathrm{C} 22 \mathrm{C}$ & $120.61(19)$ \\
\hline $\mathrm{C} 24 \mathrm{C}-\mathrm{C} 23 \mathrm{C}-\mathrm{H} 15 \mathrm{C}$ & 119.7 \\
\hline $\mathrm{C} 22 \mathrm{C}-\mathrm{C} 23 \mathrm{C}-\mathrm{H} 15 \mathrm{C}$ & 119.7 \\
\hline $\mathrm{C} 25 \mathrm{~A}-\mathrm{C} 24 \mathrm{~A}-\mathrm{C} 23 \mathrm{~A}$ & $120.6(2)$ \\
\hline $\mathrm{C} 25 \mathrm{~A}-\mathrm{C} 24 \mathrm{~A}-\mathrm{H} 16 \mathrm{~A}$ & 119.7 \\
\hline $\mathrm{C} 23 \mathrm{~A}-\mathrm{C} 24 \mathrm{~A}-\mathrm{H} 16 \mathrm{~A}$ & 119.7 \\
\hline $\mathrm{C} 25 \mathrm{~B}-\mathrm{C} 24 \mathrm{~B}-\mathrm{C} 23 \mathrm{~B}$ & $120.9(2)$ \\
\hline $\mathrm{C} 25 \mathrm{~B}-\mathrm{C} 24 \mathrm{~B}-\mathrm{H} 16 \mathrm{~B}$ & 119.6 \\
\hline $\mathrm{C} 23 \mathrm{~B}-\mathrm{C} 24 \mathrm{~B}-\mathrm{H} 16 \mathrm{~B}$ & 119.6 \\
\hline $\mathrm{C} 25 \mathrm{C}-\mathrm{C} 24 \mathrm{C}-\mathrm{C} 23 \mathrm{C}$ & $121.4(2)$ \\
\hline $\mathrm{C} 25 \mathrm{C}-\mathrm{C} 24 \mathrm{C}-\mathrm{H} 16 \mathrm{C}$ & 119.3 \\
\hline $\mathrm{C} 23 \mathrm{C}-\mathrm{C} 24 \mathrm{C}-\mathrm{H} 16 \mathrm{C}$ & 119.3 \\
\hline $\mathrm{C} 26 \mathrm{~A}-\mathrm{C} 25 \mathrm{~A}-\mathrm{C} 24 \mathrm{~A}$ & $118.2(2)$ \\
\hline $\mathrm{C} 26 \mathrm{~A}-\mathrm{C} 25 \mathrm{~A}-\mathrm{H} 17 \mathrm{~A}$ & 120.9 \\
\hline $\mathrm{C} 24 \mathrm{~A}-\mathrm{C} 25 \mathrm{~A}-\mathrm{H} 17 \mathrm{~A}$ & 120.9 \\
\hline $\mathrm{C} 24 \mathrm{~B}-\mathrm{C} 25 \mathrm{~B}-\mathrm{C} 26 \mathrm{~B}$ & $118.7(2)$ \\
\hline $\mathrm{C} 24 \mathrm{~B}-\mathrm{C} 25 \mathrm{~B}-\mathrm{H} 17 \mathrm{~B}$ & 120.7 \\
\hline $\mathrm{C} 26 \mathrm{~B}-\mathrm{C} 25 \mathrm{~B}-\mathrm{H} 17 \mathrm{~B}$ & 120.7 \\
\hline $\mathrm{C} 24 \mathrm{C}-\mathrm{C} 25 \mathrm{C}-\mathrm{C} 26 \mathrm{C}$ & $118.6(2)$ \\
\hline $\mathrm{C} 24 \mathrm{C}-\mathrm{C} 25 \mathrm{C}-\mathrm{H} 17 \mathrm{C}$ & 120.7 \\
\hline $\mathrm{C} 26 \mathrm{C}-\mathrm{C} 25 \mathrm{C}-\mathrm{H} 17 \mathrm{C}$ & 120.7 \\
\hline $\mathrm{C} 25 \mathrm{~A}-\mathrm{C} 26 \mathrm{~A}-\mathrm{C} 27 \mathrm{~A}$ & $121.8(2)$ \\
\hline $\mathrm{C} 25 \mathrm{~A}-\mathrm{C} 26 \mathrm{~A}-\mathrm{H} 18 \mathrm{~A}$ & 119.1 \\
\hline $\mathrm{C} 27 \mathrm{~A}-\mathrm{C} 26 \mathrm{~A}-\mathrm{H} 18 \mathrm{~A}$ & 119.1 \\
\hline $\mathrm{C} 25 \mathrm{~B}-\mathrm{C} 26 \mathrm{~B}-\mathrm{C} 27 \mathrm{~B}$ & $121.2(2)$ \\
\hline
\end{tabular}




\begin{tabular}{|c|c|c|c|}
\hline $\mathrm{C} 13 \mathrm{~A}-\mathrm{C} 12 \mathrm{~A}-\mathrm{H} 9 \mathrm{~A}$ & 119.5 & $\mathrm{C} 25 \mathrm{~B}-\mathrm{C} 26 \mathrm{~B}-\mathrm{H} 18 \mathrm{~B}$ & 119.4 \\
\hline $\mathrm{C} 11 \mathrm{~B}-\mathrm{C} 12 \mathrm{~B}-\mathrm{C} 13 \mathrm{~B}$ & $121.0(2)$ & $\mathrm{C} 27 \mathrm{~B}-\mathrm{C} 26 \mathrm{~B}-\mathrm{H} 18 \mathrm{~B}$ & 119.4 \\
\hline $\mathrm{C} 11 \mathrm{~B}-\mathrm{C} 12 \mathrm{~B}-\mathrm{H} 9 \mathrm{~B}$ & 119.5 & $\mathrm{C} 27 \mathrm{C}-\mathrm{C} 26 \mathrm{C}-\mathrm{C} 25 \mathrm{C}$ & $120.5(2)$ \\
\hline $\mathrm{C} 13 \mathrm{~B}-\mathrm{C} 12 \mathrm{~B}-\mathrm{H} 9 \mathrm{~B}$ & 119.5 & $\mathrm{C} 27 \mathrm{C}-\mathrm{C} 26 \mathrm{C}-\mathrm{H} 18 \mathrm{C}$ & 119.7 \\
\hline $\mathrm{C} 11 \mathrm{C}-\mathrm{C} 12 \mathrm{C}-\mathrm{C} 13 \mathrm{C}$ & $121.4(2)$ & $\mathrm{C} 25 \mathrm{C}-\mathrm{C} 26 \mathrm{C}-\mathrm{H} 18 \mathrm{C}$ & 119.7 \\
\hline $\mathrm{C} 11 \mathrm{C}-\mathrm{C} 12 \mathrm{C}-\mathrm{H} 9 \mathrm{C}$ & 119.3 & $\mathrm{C} 22 \mathrm{~A}-\mathrm{C} 27 \mathrm{~A}-\mathrm{C} 26 \mathrm{~A}$ & $120.2(2)$ \\
\hline $\mathrm{C} 13 \mathrm{C}-\mathrm{C} 12 \mathrm{C}-\mathrm{H} 9 \mathrm{C}$ & 119.3 & $\mathrm{C} 22 \mathrm{~A}-\mathrm{C} 27 \mathrm{~A}-\mathrm{H} 19 \mathrm{~A}$ & 119.9 \\
\hline $\mathrm{C} 14 \mathrm{~A}-\mathrm{C} 13 \mathrm{~A}-\mathrm{C} 12 \mathrm{~A}$ & $123.68(19)$ & $\mathrm{C} 26 \mathrm{~A}-\mathrm{C} 27 \mathrm{~A}-\mathrm{H} 19 \mathrm{~A}$ & 119.9 \\
\hline $\mathrm{C} 14 \mathrm{~A}-\mathrm{C} 13 \mathrm{~A}-\mathrm{C} 8 \mathrm{~A}$ & $118.98(19)$ & $\mathrm{C} 26 \mathrm{~B}-\mathrm{C} 27 \mathrm{~B}-\mathrm{C} 22 \mathrm{~B}$ & $120.4(2)$ \\
\hline $\mathrm{C} 12 \mathrm{~A}-\mathrm{C} 13 \mathrm{~A}-\mathrm{C} 8 \mathrm{~A}$ & $117.34(19)$ & $\mathrm{C} 26 \mathrm{~B}-\mathrm{C} 27 \mathrm{~B}-\mathrm{H} 19 \mathrm{~B}$ & 119.8 \\
\hline $\mathrm{C} 14 \mathrm{~B}-\mathrm{C} 13 \mathrm{~B}-\mathrm{C} 12 \mathrm{~B}$ & $123.45(18)$ & $\mathrm{C} 22 \mathrm{~B}-\mathrm{C} 27 \mathrm{~B}-\mathrm{H} 19 \mathrm{~B}$ & 119.8 \\
\hline $\mathrm{C} 14 \mathrm{~B}-\mathrm{C} 13 \mathrm{~B}-\mathrm{C} 8 \mathrm{~B}$ & $119.23(19)$ & $\mathrm{C} 26 \mathrm{C}-\mathrm{C} 27 \mathrm{C}-\mathrm{C} 22 \mathrm{C}$ & $121.4(2)$ \\
\hline $\mathrm{C} 12 \mathrm{~B}-\mathrm{C} 13 \mathrm{~B}-\mathrm{C} 8 \mathrm{~B}$ & $117.29(18)$ & $\mathrm{C} 26 \mathrm{C}-\mathrm{C} 27 \mathrm{C}-\mathrm{H} 19 \mathrm{C}$ & 119.3 \\
\hline $\mathrm{C} 14 \mathrm{C}-\mathrm{C} 13 \mathrm{C}-\mathrm{C} 12 \mathrm{C}$ & $123.42(19)$ & $\mathrm{C} 22 \mathrm{C}-\mathrm{C} 27 \mathrm{C}-\mathrm{H} 19 \mathrm{C}$ & 119.3 \\
\hline $\mathrm{C} 14 \mathrm{C}-\mathrm{C} 13 \mathrm{C}-\mathrm{C} 8 \mathrm{C}$ & $118.7(2)$ & $\mathrm{C} 15 \mathrm{~A}-\mathrm{N} 1 \mathrm{~A}-\mathrm{C} 16 \mathrm{~A}$ & $117.97(16)$ \\
\hline $\mathrm{C} 12 \mathrm{C}-\mathrm{C} 13 \mathrm{C}-\mathrm{C} 8 \mathrm{C}$ & $117.7(2)$ & $\mathrm{C} 15 \mathrm{~B}-\mathrm{N} 1 \mathrm{~B}-\mathrm{C} 16 \mathrm{~B}$ & $119.33(16)$ \\
\hline $\mathrm{C} 13 \mathrm{~A}-\mathrm{C} 14 \mathrm{~A}-\mathrm{C} 1 \mathrm{~A}$ & $120.68(18)$ & $\mathrm{C} 15 \mathrm{C}-\mathrm{N} 1 \mathrm{C}-\mathrm{C} 16 \mathrm{C}$ & $119.17(16)$ \\
\hline $\mathrm{C} 13 \mathrm{~A}-\mathrm{C} 14 \mathrm{~A}-\mathrm{C} 15 \mathrm{~A}$ & $121.49(18)$ & $\mathrm{C} 19 \mathrm{~A}-\mathrm{N} 2 \mathrm{~A}-\mathrm{C} 22 \mathrm{~A}$ & $130.68(17)$ \\
\hline $\mathrm{C} 1 \mathrm{~A}-\mathrm{C} 14 \mathrm{~A}-\mathrm{C} 15 \mathrm{~A}$ & $117.74(17)$ & $\mathrm{C} 19 \mathrm{~A}-\mathrm{N} 2 \mathrm{~A}-\mathrm{H} 20 \mathrm{~A}$ & $114.2(14)$ \\
\hline $\mathrm{C} 13 \mathrm{~B}-\mathrm{C} 14 \mathrm{~B}-\mathrm{C} 1 \mathrm{~B}$ & $120.57(18)$ & $\mathrm{C} 22 \mathrm{~A}-\mathrm{N} 2 \mathrm{~A}-\mathrm{H} 20 \mathrm{~A}$ & $114.1(13)$ \\
\hline $\mathrm{C} 13 \mathrm{~B}-\mathrm{C} 14 \mathrm{~B}-\mathrm{C} 15 \mathrm{~B}$ & $121.34(18)$ & $\mathrm{C} 19 \mathrm{~B}-\mathrm{N} 2 \mathrm{~B}-\mathrm{C} 22 \mathrm{~B}$ & $130.67(17)$ \\
\hline $\mathrm{C} 1 \mathrm{~B}-\mathrm{C} 14 \mathrm{~B}-\mathrm{C} 15 \mathrm{~B}$ & $117.97(18)$ & $\mathrm{C} 19 \mathrm{~B}-\mathrm{N} 2 \mathrm{~B}-\mathrm{H} 20 \mathrm{~B}$ & $114.1(14)$ \\
\hline $\mathrm{C} 1 \mathrm{C}-\mathrm{C} 14 \mathrm{C}-\mathrm{C} 13 \mathrm{C}$ & $121.10(19)$ & $\mathrm{C} 22 \mathrm{~B}-\mathrm{N} 2 \mathrm{~B}-\mathrm{H} 20 \mathrm{~B}$ & $114.5(14)$ \\
\hline $\mathrm{C} 1 \mathrm{C}-\mathrm{C} 14 \mathrm{C}-\mathrm{C} 15 \mathrm{C}$ & $120.96(19)$ & $\mathrm{C} 19 \mathrm{C}-\mathrm{N} 2 \mathrm{C}-\mathrm{C} 22 \mathrm{C}$ & $132.68(17)$ \\
\hline $\mathrm{C} 13 \mathrm{C}-\mathrm{C} 14 \mathrm{C}-\mathrm{C} 15 \mathrm{C}$ & $117.76(19)$ & $\mathrm{C} 19 \mathrm{C}-\mathrm{N} 2 \mathrm{C}-\mathrm{H} 20 \mathrm{C}$ & $112.0(12)$ \\
\hline $\mathrm{N} 1 \mathrm{~A}-\mathrm{C} 15 \mathrm{~A}-\mathrm{C} 14 \mathrm{~A}$ & $122.92(18)$ & $\mathrm{C} 22 \mathrm{C}-\mathrm{N} 2 \mathrm{C}-\mathrm{H} 20 \mathrm{C}$ & $115.3(12)$ \\
\hline $\mathrm{N} 1 \mathrm{~A}-\mathrm{C} 15 \mathrm{~A}-\mathrm{H} 10 \mathrm{~A}$ & 118.5 & & \\
\hline $\mathrm{C} 14 \mathrm{~A}-\mathrm{C} 1 \mathrm{~A}-\mathrm{C} 2 \mathrm{~A}-\mathrm{C} 3 \mathrm{~A}$ & $179.77(19)$ & $\mathrm{C} 2 \mathrm{~B}-\mathrm{C} 1 \mathrm{~B}-\mathrm{C} 14 \mathrm{~B}-\mathrm{C} 15 \mathrm{~B}$ & $2.5(3)$ \\
\hline $\mathrm{C} 6 \mathrm{~A}-\mathrm{C} 1 \mathrm{~A}-\mathrm{C} 2 \mathrm{~A}-\mathrm{C} 3 \mathrm{~A}$ & $-3.1(3)$ & $\mathrm{C} 6 \mathrm{~B}-\mathrm{C} 1 \mathrm{~B}-\mathrm{C} 14 \mathrm{~B}-\mathrm{C} 15 \mathrm{~B}$ & $-172.84(17)$ \\
\hline $\mathrm{C} 14 \mathrm{~B}-\mathrm{C} 1 \mathrm{~B}-\mathrm{C} 2 \mathrm{~B}-\mathrm{C} 3 \mathrm{~B}$ & $-176.94(19)$ & $\mathrm{C} 2 \mathrm{C}-\mathrm{C} 1 \mathrm{C}-\mathrm{C} 14 \mathrm{C}-\mathrm{C} 13 \mathrm{C}$ & $-177.79(18)$ \\
\hline $\mathrm{C} 6 \mathrm{~B}-\mathrm{C} 1 \mathrm{~B}-\mathrm{C} 2 \mathrm{~B}-\mathrm{C} 3 \mathrm{~B}$ & $-1.5(3)$ & $\mathrm{C} 6 \mathrm{C}-\mathrm{C} 1 \mathrm{C}-\mathrm{C} 14 \mathrm{C}-\mathrm{C} 13 \mathrm{C}$ & $0.9(3)$ \\
\hline $\mathrm{C} 14 \mathrm{C}-\mathrm{C} 1 \mathrm{C}-\mathrm{C} 2 \mathrm{C}-\mathrm{C} 3 \mathrm{C}$ & $179.74(19)$ & $\mathrm{C} 2 \mathrm{C}-\mathrm{C} 1 \mathrm{C}-\mathrm{C} 14 \mathrm{C}-\mathrm{C} 15 \mathrm{C}$ & $7.2(3)$ \\
\hline $\mathrm{C} 6 \mathrm{C}-\mathrm{C} 1 \mathrm{C}-\mathrm{C} 2 \mathrm{C}-\mathrm{C} 3 \mathrm{C}$ & $1.0(3)$ & $\mathrm{C} 6 \mathrm{C}-\mathrm{C} 1 \mathrm{C}-\mathrm{C} 14 \mathrm{C}-\mathrm{C} 15 \mathrm{C}$ & $-174.09(18)$ \\
\hline $\mathrm{C} 1 \mathrm{~A}-\mathrm{C} 2 \mathrm{~A}-\mathrm{C} 3 \mathrm{~A}-\mathrm{C} 4 \mathrm{~A}$ & $0.8(3)$ & $\mathrm{C} 12 \mathrm{C}-\mathrm{C} 13 \mathrm{C}-\mathrm{C} 14 \mathrm{C}-\mathrm{C} 1 \mathrm{C}$ & $-176.86(19)$ \\
\hline $\mathrm{C} 1 \mathrm{~B}-\mathrm{C} 2 \mathrm{~B}-\mathrm{C} 3 \mathrm{~B}-\mathrm{C} 4 \mathrm{~B}$ & $1.0(3)$ & $\mathrm{C} 8 \mathrm{C}-\mathrm{C} 13 \mathrm{C}-\mathrm{C} 14 \mathrm{C}-\mathrm{C} 1 \mathrm{C}$ & $-0.5(3)$ \\
\hline $\mathrm{C} 1 \mathrm{C}-\mathrm{C} 2 \mathrm{C}-\mathrm{C} 3 \mathrm{C}-\mathrm{C} 4 \mathrm{C}$ & $-0.8(3)$ & $\mathrm{C} 12 \mathrm{C}-\mathrm{C} 13 \mathrm{C}-\mathrm{C} 14 \mathrm{C}-\mathrm{C} 15 \mathrm{C}$ & $-1.7(3)$ \\
\hline $\mathrm{C} 2 \mathrm{~A}-\mathrm{C} 3 \mathrm{~A}-\mathrm{C} 4 \mathrm{~A}-\mathrm{C} 5 \mathrm{~A}$ & $1.6(3)$ & $\mathrm{C} 8 \mathrm{C}-\mathrm{C} 13 \mathrm{C}-\mathrm{C} 14 \mathrm{C}-\mathrm{C} 15 \mathrm{C}$ & $174.68(17)$ \\
\hline $\mathrm{C} 2 \mathrm{~B}-\mathrm{C} 3 \mathrm{~B}-\mathrm{C} 4 \mathrm{~B}-\mathrm{C} 5 \mathrm{~B}$ & $0.0(3)$ & $\mathrm{C} 13 \mathrm{~A}-\mathrm{C} 14 \mathrm{~A}-\mathrm{C} 15 \mathrm{~A}-\mathrm{N} 1 \mathrm{~A}$ & $-41.9(3)$ \\
\hline $\mathrm{C} 2 \mathrm{C}-\mathrm{C} 3 \mathrm{C}-\mathrm{C} 4 \mathrm{C}-\mathrm{C} 5 \mathrm{C}$ & $0.1(3)$ & $\mathrm{C} 1 \mathrm{~A}-\mathrm{C} 14 \mathrm{~A}-\mathrm{C} 15 \mathrm{~A}-\mathrm{N} 1 \mathrm{~A}$ & $134.6(2)$ \\
\hline $\mathrm{C} 3 \mathrm{~A}-\mathrm{C} 4 \mathrm{~A}-\mathrm{C} 5 \mathrm{~A}-\mathrm{C} 6 \mathrm{~A}$ & $-1.4(3)$ & $\mathrm{C} 13 \mathrm{~B}-\mathrm{C} 14 \mathrm{~B}-\mathrm{C} 15 \mathrm{~B}-\mathrm{N} 1 \mathrm{~B}$ & $-44.8(3)$ \\
\hline $\mathrm{C} 3 \mathrm{~B}-\mathrm{C} 4 \mathrm{~B}-\mathrm{C} 5 \mathrm{~B}-\mathrm{C} 6 \mathrm{~B}$ & $-0.4(3)$ & $\mathrm{C} 1 \mathrm{~B}-\mathrm{C} 14 \mathrm{~B}-\mathrm{C} 15 \mathrm{~B}-\mathrm{N} 1 \mathrm{~B}$ & $131.35(19)$ \\
\hline $\mathrm{C} 3 \mathrm{C}-\mathrm{C} 4 \mathrm{C}-\mathrm{C} 5 \mathrm{C}-\mathrm{C} 6 \mathrm{C}$ & $0.4(3)$ & $\mathrm{C} 1 \mathrm{C}-\mathrm{C} 14 \mathrm{C}-\mathrm{C} 15 \mathrm{C}-\mathrm{N} 1 \mathrm{C}$ & $48.9(3)$ \\
\hline $\mathrm{C} 4 \mathrm{~A}-\mathrm{C} 5 \mathrm{~A}-\mathrm{C} 6 \mathrm{~A}-\mathrm{C} 7 \mathrm{~A}$ & $177.4(2)$ & $\mathrm{C} 13 \mathrm{C}-\mathrm{C} 14 \mathrm{C}-\mathrm{C} 15 \mathrm{C}-\mathrm{N} 1 \mathrm{C}$ & $-126.3(2)$ \\
\hline $\mathrm{C} 4 \mathrm{~A}-\mathrm{C} 5 \mathrm{~A}-\mathrm{C} 6 \mathrm{~A}-\mathrm{C} 1 \mathrm{~A}$ & $-0.9(3)$ & $\mathrm{C} 21 \mathrm{~A}-\mathrm{C} 16 \mathrm{~A}-\mathrm{C} 17 \mathrm{~A}-\mathrm{C} 18 \mathrm{~A}$ & $-4.9(3)$ \\
\hline $\mathrm{C} 14 \mathrm{~A}-\mathrm{C} 1 \mathrm{~A}-\mathrm{C} 6 \mathrm{~A}-\mathrm{C} 7 \mathrm{~A}$ & $2.0(3)$ & $\mathrm{N} 1 \mathrm{~A}-\mathrm{C} 16 \mathrm{~A}-\mathrm{C} 17 \mathrm{~A}-\mathrm{C} 18 \mathrm{~A}$ & $175.18(17)$ \\
\hline $\mathrm{C} 2 \mathrm{~A}-\mathrm{C} 1 \mathrm{~A}-\mathrm{C} 6 \mathrm{~A}-\mathrm{C} 7 \mathrm{~A}$ & $-175.28(18)$ & $\mathrm{C} 21 \mathrm{~B}-\mathrm{C} 16 \mathrm{~B}-\mathrm{C} 17 \mathrm{~B}-\mathrm{C} 18 \mathrm{~B}$ & $-5.6(3)$ \\
\hline $\mathrm{C} 14 \mathrm{~A}-\mathrm{C} 1 \mathrm{~A}-\mathrm{C} 6 \mathrm{~A}-\mathrm{C} 5 \mathrm{~A}$ & $-179.59(17)$ & $\mathrm{N} 1 \mathrm{~B}-\mathrm{C} 16 \mathrm{~B}-\mathrm{C} 17 \mathrm{~B}-\mathrm{C} 18 \mathrm{~B}$ & $173.92(17)$ \\
\hline
\end{tabular}




\begin{tabular}{|c|c|c|c|}
\hline $\mathrm{C} 2 \mathrm{~A}-\mathrm{C} 1 \mathrm{~A}-\mathrm{C} 6 \mathrm{~A}-\mathrm{C} 5 \mathrm{~A}$ & $3.1(3)$ & $\mathrm{C} 21 \mathrm{C}-\mathrm{C} 16 \mathrm{C}-\mathrm{C} 17 \mathrm{C}-\mathrm{C} 18 \mathrm{C}$ & $-4.0(3)$ \\
\hline $\mathrm{C} 4 \mathrm{~B}-\mathrm{C} 5 \mathrm{~B}-\mathrm{C} 6 \mathrm{~B}-\mathrm{C} 7 \mathrm{~B}$ & $178.4(2)$ & $\mathrm{N} 1 \mathrm{C}-\mathrm{C} 16 \mathrm{C}-\mathrm{C} 17 \mathrm{C}-\mathrm{C} 18 \mathrm{C}$ & $175.62(17)$ \\
\hline $\mathrm{C} 4 \mathrm{~B}-\mathrm{C} 5 \mathrm{~B}-\mathrm{C} 6 \mathrm{~B}-\mathrm{C} 1 \mathrm{~B}$ & $-0.1(3)$ & $\mathrm{C} 16 \mathrm{~A}-\mathrm{C} 17 \mathrm{~A}-\mathrm{C} 18 \mathrm{~A}-\mathrm{C} 19 \mathrm{~A}$ & $-0.3(3)$ \\
\hline $\mathrm{C} 14 \mathrm{~B}-\mathrm{C} 1 \mathrm{~B}-\mathrm{C} 6 \mathrm{~B}-\mathrm{C} 7 \mathrm{~B}$ & $-1.9(3)$ & $\mathrm{C} 16 \mathrm{~B}-\mathrm{C} 17 \mathrm{~B}-\mathrm{C} 18 \mathrm{~B}-\mathrm{C} 19 \mathrm{~B}$ & $0.4(3)$ \\
\hline $\mathrm{C} 2 \mathrm{~B}-\mathrm{C} 1 \mathrm{~B}-\mathrm{C} 6 \mathrm{~B}-\mathrm{C} 7 \mathrm{~B}$ & $-177.54(18)$ & $\mathrm{C} 16 \mathrm{C}-\mathrm{C} 17 \mathrm{C}-\mathrm{C} 18 \mathrm{C}-\mathrm{C} 19 \mathrm{C}$ & $3.3(3)$ \\
\hline $\mathrm{C} 14 \mathrm{~B}-\mathrm{C} 1 \mathrm{~B}-\mathrm{C} 6 \mathrm{~B}-\mathrm{C} 5 \mathrm{~B}$ & $176.68(18)$ & $\mathrm{C} 17 \mathrm{~A}-\mathrm{C} 18 \mathrm{~A}-\mathrm{C} 19 \mathrm{~A}-\mathrm{N} 2 \mathrm{~A}$ & $-178.59(17)$ \\
\hline $\mathrm{C} 2 \mathrm{~B}-\mathrm{C} 1 \mathrm{~B}-\mathrm{C} 6 \mathrm{~B}-\mathrm{C} 5 \mathrm{~B}$ & $1.0(3)$ & $\mathrm{C} 17 \mathrm{~A}-\mathrm{C} 18 \mathrm{~A}-\mathrm{C} 19 \mathrm{~A}-\mathrm{C} 20 \mathrm{~A}$ & $4.9(3)$ \\
\hline $\mathrm{C} 4 \mathrm{C}-\mathrm{C} 5 \mathrm{C}-\mathrm{C} 6 \mathrm{C}-\mathrm{C} 7 \mathrm{C}$ & $-179.1(2)$ & $\mathrm{C} 17 \mathrm{~B}-\mathrm{C} 18 \mathrm{~B}-\mathrm{C} 19 \mathrm{~B}-\mathrm{N} 2 \mathrm{~B}$ & $-178.26(17)$ \\
\hline $\mathrm{C} 4 \mathrm{C}-\mathrm{C} 5 \mathrm{C}-\mathrm{C} 6 \mathrm{C}-\mathrm{C} 1 \mathrm{C}$ & $-0.2(3)$ & $\mathrm{C} 17 \mathrm{~B}-\mathrm{C} 18 \mathrm{~B}-\mathrm{C} 19 \mathrm{~B}-\mathrm{C} 20 \mathrm{~B}$ & $5.1(3)$ \\
\hline $\mathrm{C} 14 \mathrm{C}-\mathrm{C} 1 \mathrm{C}-\mathrm{C} 6 \mathrm{C}-\mathrm{C} 7 \mathrm{C}$ & $-0.4(3)$ & $\mathrm{C} 17 \mathrm{C}-\mathrm{C} 18 \mathrm{C}-\mathrm{C} 19 \mathrm{C}-\mathrm{N} 2 \mathrm{C}$ & $-178.85(18)$ \\
\hline $\mathrm{C} 2 \mathrm{C}-\mathrm{C} 1 \mathrm{C}-\mathrm{C} 6 \mathrm{C}-\mathrm{C} 7 \mathrm{C}$ & $178.43(18)$ & $\mathrm{C} 17 \mathrm{C}-\mathrm{C} 18 \mathrm{C}-\mathrm{C} 19 \mathrm{C}-\mathrm{C} 20 \mathrm{C}$ & $-0.4(3)$ \\
\hline $\mathrm{C} 14 \mathrm{C}-\mathrm{C} 1 \mathrm{C}-\mathrm{C} 6 \mathrm{C}-\mathrm{C} 5 \mathrm{C}$ & $-179.29(18)$ & $\mathrm{N} 2 \mathrm{~A}-\mathrm{C} 19 \mathrm{~A}-\mathrm{C} 20 \mathrm{~A}-\mathrm{C} 21 \mathrm{~A}$ & $179.34(18)$ \\
\hline $\mathrm{C} 2 \mathrm{C}-\mathrm{C} 1 \mathrm{C}-\mathrm{C} 6 \mathrm{C}-\mathrm{C} 5 \mathrm{C}$ & $-0.5(3)$ & $\mathrm{C} 18 \mathrm{~A}-\mathrm{C} 19 \mathrm{~A}-\mathrm{C} 20 \mathrm{~A}-\mathrm{C} 21 \mathrm{~A}$ & $-4.4(3)$ \\
\hline $\mathrm{C} 5 \mathrm{~A}-\mathrm{C} 6 \mathrm{~A}-\mathrm{C} 7 \mathrm{~A}-\mathrm{C} 8 \mathrm{~A}$ & $179.69(19)$ & $\mathrm{N} 2 \mathrm{~B}-\mathrm{C} 19 \mathrm{~B}-\mathrm{C} 20 \mathrm{~B}-\mathrm{C} 21 \mathrm{~B}$ & 178.23 (19) \\
\hline $\mathrm{C} 1 \mathrm{~A}-\mathrm{C} 6 \mathrm{~A}-\mathrm{C} 7 \mathrm{~A}-\mathrm{C} 8 \mathrm{~A}$ & $-2.0(3)$ & $\mathrm{C} 18 \mathrm{~B}-\mathrm{C} 19 \mathrm{~B}-\mathrm{C} 20 \mathrm{~B}-\mathrm{C} 21 \mathrm{~B}$ & $-5.4(3)$ \\
\hline $\mathrm{C} 5 \mathrm{~B}-\mathrm{C} 6 \mathrm{~B}-\mathrm{C} 7 \mathrm{~B}-\mathrm{C} 8 \mathrm{~B}$ & $-178.80(19)$ & $\mathrm{N} 2 \mathrm{C}-\mathrm{C} 19 \mathrm{C}-\mathrm{C} 20 \mathrm{C}-\mathrm{C} 21 \mathrm{C}$ & $176.77(17)$ \\
\hline $\mathrm{C} 1 \mathrm{~B}-\mathrm{C} 6 \mathrm{~B}-\mathrm{C} 7 \mathrm{~B}-\mathrm{C} 8 \mathrm{~B}$ & $-0.3(3)$ & $\mathrm{C} 18 \mathrm{C}-\mathrm{C} 19 \mathrm{C}-\mathrm{C} 20 \mathrm{C}-\mathrm{C} 21 \mathrm{C}$ & $-1.9(3)$ \\
\hline $\mathrm{C} 5 \mathrm{C}-\mathrm{C} 6 \mathrm{C}-\mathrm{C} 7 \mathrm{C}-\mathrm{C} 8 \mathrm{C}$ & $178.2(2)$ & $\mathrm{C} 17 \mathrm{~A}-\mathrm{C} 16 \mathrm{~A}-\mathrm{C} 21 \mathrm{~A}-\mathrm{C} 20 \mathrm{~A}$ & $5.3(3)$ \\
\hline $\mathrm{C} 1 \mathrm{C}-\mathrm{C} 6 \mathrm{C}-\mathrm{C} 7 \mathrm{C}-\mathrm{C} 8 \mathrm{C}$ & $-0.6(3)$ & $\mathrm{N} 1 \mathrm{~A}-\mathrm{C} 16 \mathrm{~A}-\mathrm{C} 21 \mathrm{~A}-\mathrm{C} 20 \mathrm{~A}$ & $-174.70(18)$ \\
\hline $\mathrm{C} 6 \mathrm{~A}-\mathrm{C} 7 \mathrm{~A}-\mathrm{C} 8 \mathrm{~A}-\mathrm{C} 9 \mathrm{~A}$ & $-179.57(19)$ & $\mathrm{C} 19 \mathrm{~A}-\mathrm{C} 20 \mathrm{~A}-\mathrm{C} 21 \mathrm{~A}-\mathrm{C} 16 \mathrm{~A}$ & $-0.7(3)$ \\
\hline $\mathrm{C} 6 \mathrm{~A}-\mathrm{C} 7 \mathrm{~A}-\mathrm{C} 8 \mathrm{~A}-\mathrm{C} 13 \mathrm{~A}$ & $-0.1(3)$ & $\mathrm{C} 19 \mathrm{~B}-\mathrm{C} 20 \mathrm{~B}-\mathrm{C} 21 \mathrm{~B}-\mathrm{C} 16 \mathrm{~B}$ & $0.3(3)$ \\
\hline $\mathrm{C} 6 \mathrm{~B}-\mathrm{C} 7 \mathrm{~B}-\mathrm{C} 8 \mathrm{~B}-\mathrm{C} 9 \mathrm{~B}$ & $-177.34(19)$ & $\mathrm{C} 17 \mathrm{~B}-\mathrm{C} 16 \mathrm{~B}-\mathrm{C} 21 \mathrm{~B}-\mathrm{C} 20 \mathrm{~B}$ & $5.3(3)$ \\
\hline $\mathrm{C} 6 \mathrm{~B}-\mathrm{C} 7 \mathrm{~B}-\mathrm{C} 8 \mathrm{~B}-\mathrm{C} 13 \mathrm{~B}$ & $0.9(3)$ & $\mathrm{N} 1 \mathrm{~B}-\mathrm{C} 16 \mathrm{~B}-\mathrm{C} 21 \mathrm{~B}-\mathrm{C} 20 \mathrm{~B}$ & $-174.25(18)$ \\
\hline $\mathrm{C} 6 \mathrm{C}-\mathrm{C} 7 \mathrm{C}-\mathrm{C} 8 \mathrm{C}-\mathrm{C} 9 \mathrm{C}$ & $178.9(2)$ & $\mathrm{C} 19 \mathrm{C}-\mathrm{C} 20 \mathrm{C}-\mathrm{C} 21 \mathrm{C}-\mathrm{C} 16 \mathrm{C}$ & $1.2(3)$ \\
\hline $\mathrm{C} 6 \mathrm{C}-\mathrm{C} 7 \mathrm{C}-\mathrm{C} 8 \mathrm{C}-\mathrm{C} 13 \mathrm{C}$ & $1.1(3)$ & $\mathrm{C} 17 \mathrm{C}-\mathrm{C} 16 \mathrm{C}-\mathrm{C} 21 \mathrm{C}-\mathrm{C} 20 \mathrm{C}$ & $1.7(3)$ \\
\hline $\mathrm{C} 7 \mathrm{~A}-\mathrm{C} 8 \mathrm{~A}-\mathrm{C} 9 \mathrm{~A}-\mathrm{C} 10 \mathrm{~A}$ & $177.7(2)$ & $\mathrm{N} 1 \mathrm{C}-\mathrm{C} 16 \mathrm{C}-\mathrm{C} 21 \mathrm{C}-\mathrm{C} 20 \mathrm{C}$ & $-177.85(17)$ \\
\hline $\mathrm{C} 13 \mathrm{~A}-\mathrm{C} 8 \mathrm{~A}-\mathrm{C} 9 \mathrm{~A}-\mathrm{C} 10 \mathrm{~A}$ & $-1.7(3)$ & $\mathrm{C} 27 \mathrm{~A}-\mathrm{C} 22 \mathrm{~A}-\mathrm{C} 23 \mathrm{~A}-\mathrm{C} 24 \mathrm{~A}$ & $0.2(3)$ \\
\hline $\mathrm{C} 7 \mathrm{~B}-\mathrm{C} 8 \mathrm{~B}-\mathrm{C} 9 \mathrm{~B}-\mathrm{C} 10 \mathrm{~B}$ & $177.1(2)$ & $\mathrm{N} 2 \mathrm{~A}-\mathrm{C} 22 \mathrm{~A}-\mathrm{C} 23 \mathrm{~A}-\mathrm{C} 24 \mathrm{~A}$ & $178.4(2)$ \\
\hline $\mathrm{C} 13 \mathrm{~B}-\mathrm{C} 8 \mathrm{~B}-\mathrm{C} 9 \mathrm{~B}-\mathrm{C} 10 \mathrm{~B}$ & $-1.2(3)$ & $\mathrm{C} 27 \mathrm{~B}-\mathrm{C} 22 \mathrm{~B}-\mathrm{C} 23 \mathrm{~B}-\mathrm{C} 24 \mathrm{~B}$ & $1.2(3)$ \\
\hline $\mathrm{C} 7 \mathrm{C}-\mathrm{C} 8 \mathrm{C}-\mathrm{C} 9 \mathrm{C}-\mathrm{C} 10 \mathrm{C}$ & $-177.4(2)$ & $\mathrm{N} 2 \mathrm{~B}-\mathrm{C} 22 \mathrm{~B}-\mathrm{C} 23 \mathrm{~B}-\mathrm{C} 24 \mathrm{~B}$ & $178.7(2)$ \\
\hline $\mathrm{C} 13 \mathrm{C}-\mathrm{C} 8 \mathrm{C}-\mathrm{C} 9 \mathrm{C}-\mathrm{C} 10 \mathrm{C}$ & $0.5(3)$ & $\mathrm{N} 2 \mathrm{C}-\mathrm{C} 22 \mathrm{C}-\mathrm{C} 23 \mathrm{C}-\mathrm{C} 24 \mathrm{C}$ & $-178.25(19)$ \\
\hline $\mathrm{C} 8 \mathrm{~A}-\mathrm{C} 9 \mathrm{~A}-\mathrm{C} 10 \mathrm{~A}-\mathrm{C} 11 \mathrm{~A}$ & $-0.3(3)$ & $\mathrm{C} 27 \mathrm{C}-\mathrm{C} 22 \mathrm{C}-\mathrm{C} 23 \mathrm{C}-\mathrm{C} 24 \mathrm{C}$ & $0.3(3)$ \\
\hline $\mathrm{C} 8 \mathrm{~B}-\mathrm{C} 9 \mathrm{~B}-\mathrm{C} 10 \mathrm{~B}-\mathrm{C} 11 \mathrm{~B}$ & $0.3(3)$ & $\mathrm{C} 22 \mathrm{~A}-\mathrm{C} 23 \mathrm{~A}-\mathrm{C} 24 \mathrm{~A}-\mathrm{C} 25 \mathrm{~A}$ & $1.1(4)$ \\
\hline $\mathrm{C} 8 \mathrm{C}-\mathrm{C} 9 \mathrm{C}-\mathrm{C} 10 \mathrm{C}-\mathrm{C} 11 \mathrm{C}$ & $0.9(4)$ & $\mathrm{C} 22 \mathrm{~B}-\mathrm{C} 23 \mathrm{~B}-\mathrm{C} 24 \mathrm{~B}-\mathrm{C} 25 \mathrm{~B}$ & $0.3(4)$ \\
\hline $\mathrm{C} 9 \mathrm{~A}-\mathrm{C} 10 \mathrm{~A}-\mathrm{C} 11 \mathrm{~A}-\mathrm{C} 12 \mathrm{~A}$ & $1.4(3)$ & $\mathrm{C} 22 \mathrm{C}-\mathrm{C} 23 \mathrm{C}-\mathrm{C} 24 \mathrm{C}-\mathrm{C} 25 \mathrm{C}$ & $1.5(3)$ \\
\hline $\mathrm{C} 9 \mathrm{~B}-\mathrm{C} 10 \mathrm{~B}-\mathrm{C} 11 \mathrm{~B}-\mathrm{C} 12 \mathrm{~B}$ & $0.8(3)$ & $\mathrm{C} 23 \mathrm{~A}-\mathrm{C} 24 \mathrm{~A}-\mathrm{C} 25 \mathrm{~A}-\mathrm{C} 26 \mathrm{~A}$ & $-1.1(3)$ \\
\hline $\mathrm{C} 9 \mathrm{C}-\mathrm{C} 10 \mathrm{C}-\mathrm{C} 11 \mathrm{C}-\mathrm{C} 12 \mathrm{C}$ & $-0.9(4)$ & $\mathrm{C} 23 \mathrm{~B}-\mathrm{C} 24 \mathrm{~B}-\mathrm{C} 25 \mathrm{~B}-\mathrm{C} 26 \mathrm{~B}$ & $-1.1(4)$ \\
\hline $\mathrm{C} 10 \mathrm{~A}-\mathrm{C} 11 \mathrm{~A}-\mathrm{C} 12 \mathrm{~A}-\mathrm{C} 13 \mathrm{~A}$ & $-0.4(3)$ & $\mathrm{C} 23 \mathrm{C}-\mathrm{C} 24 \mathrm{C}-\mathrm{C} 25 \mathrm{C}-\mathrm{C} 26 \mathrm{C}$ & $-1.8(3)$ \\
\hline $\mathrm{C} 10 \mathrm{~B}-\mathrm{C} 11 \mathrm{~B}-\mathrm{C} 12 \mathrm{~B}-\mathrm{C} 13 \mathrm{~B}$ & $-1.0(3)$ & $\mathrm{C} 24 \mathrm{~A}-\mathrm{C} 25 \mathrm{~A}-\mathrm{C} 26 \mathrm{~A}-\mathrm{C} 27 \mathrm{~A}$ & $-0.3(3)$ \\
\hline $\mathrm{C} 10 \mathrm{C}-\mathrm{C} 11 \mathrm{C}-\mathrm{C} 12 \mathrm{C}-\mathrm{C} 13 \mathrm{C}$ & $-0.6(3)$ & $\mathrm{C} 24 \mathrm{~B}-\mathrm{C} 25 \mathrm{~B}-\mathrm{C} 26 \mathrm{~B}-\mathrm{C} 27 \mathrm{~B}$ & $0.6(3)$ \\
\hline $\mathrm{C} 11 \mathrm{~A}-\mathrm{C} 12 \mathrm{~A}-\mathrm{C} 13 \mathrm{~A}-\mathrm{C} 14 \mathrm{~A}$ & 179.49 (19) & $\mathrm{C} 24 \mathrm{C}-\mathrm{C} 25 \mathrm{C}-\mathrm{C} 26 \mathrm{C}-\mathrm{C} 27 \mathrm{C}$ & $0.3(3)$ \\
\hline $\mathrm{C} 11 \mathrm{~A}-\mathrm{C} 12 \mathrm{~A}-\mathrm{C} 13 \mathrm{~A}-\mathrm{C} 8 \mathrm{~A}$ & $-1.5(3)$ & $\mathrm{C} 23 \mathrm{~A}-\mathrm{C} 22 \mathrm{~A}-\mathrm{C} 27 \mathrm{~A}-\mathrm{C} 26 \mathrm{~A}$ & $-1.6(3)$ \\
\hline $\mathrm{C} 7 \mathrm{~A}-\mathrm{C} 8 \mathrm{~A}-\mathrm{C} 13 \mathrm{~A}-\mathrm{C} 14 \mathrm{~A}$ & $2.2(3)$ & $\mathrm{N} 2 \mathrm{~A}-\mathrm{C} 22 \mathrm{~A}-\mathrm{C} 27 \mathrm{~A}-\mathrm{C} 26 \mathrm{~A}$ & $-179.6(2)$ \\
\hline $\mathrm{C} 9 \mathrm{~A}-\mathrm{C} 8 \mathrm{~A}-\mathrm{C} 13 \mathrm{~A}-\mathrm{C} 14 \mathrm{~A}$ & $-178.39(18)$ & $\mathrm{C} 25 \mathrm{~A}-\mathrm{C} 26 \mathrm{~A}-\mathrm{C} 27 \mathrm{~A}-\mathrm{C} 22 \mathrm{~A}$ & $1.7(3)$ \\
\hline $\mathrm{C} 7 \mathrm{~A}-\mathrm{C} 8 \mathrm{~A}-\mathrm{C} 13 \mathrm{~A}-\mathrm{C} 12 \mathrm{~A}$ & $-176.89(18)$ & $\mathrm{C} 25 \mathrm{~B}-\mathrm{C} 26 \mathrm{~B}-\mathrm{C} 27 \mathrm{~B}-\mathrm{C} 22 \mathrm{~B}$ & $0.9(3)$ \\
\hline $\mathrm{C} 9 \mathrm{~A}-\mathrm{C} 8 \mathrm{~A}-\mathrm{C} 13 \mathrm{~A}-\mathrm{C} 12 \mathrm{~A}$ & $2.6(3)$ & $\mathrm{C} 23 \mathrm{~B}-\mathrm{C} 22 \mathrm{~B}-\mathrm{C} 27 \mathrm{~B}-\mathrm{C} 26 \mathrm{~B}$ & $-1.7(3)$ \\
\hline $\mathrm{C} 11 \mathrm{~B}-\mathrm{C} 12 \mathrm{~B}-\mathrm{C} 13 \mathrm{~B}-\mathrm{C} 14 \mathrm{~B}$ & $-177.72(19)$ & $\mathrm{N} 2 \mathrm{~B}-\mathrm{C} 22 \mathrm{~B}-\mathrm{C} 27 \mathrm{~B}-\mathrm{C} 26 \mathrm{~B}$ & $-179.06(19)$ \\
\hline $\mathrm{C} 11 \mathrm{~B}-\mathrm{C} 12 \mathrm{~B}-\mathrm{C} 13 \mathrm{~B}-\mathrm{C} 8 \mathrm{~B}$ & $0.1(3)$ & $\mathrm{C} 25 \mathrm{C}-\mathrm{C} 26 \mathrm{C}-\mathrm{C} 27 \mathrm{C}-\mathrm{C} 22 \mathrm{C}$ & $1.4(3)$ \\
\hline
\end{tabular}




$\begin{array}{ll}\mathrm{C} 7 \mathrm{~B}-\mathrm{C} 8 \mathrm{~B}-\mathrm{C} 13 \mathrm{~B}-\mathrm{C} 14 \mathrm{~B} & 0.6(3) \\ \mathrm{C} 9 \mathrm{~B}-\mathrm{C} 8 \mathrm{~B}-\mathrm{C} 13 \mathrm{~B}-\mathrm{C} 14 \mathrm{~B} & 178.89(17) \\ \mathrm{C} 7 \mathrm{~B}-\mathrm{C} 8 \mathrm{~B}-\mathrm{C} 13 \mathrm{~B}-\mathrm{C} 12 \mathrm{~B} & -177.36(17) \\ \mathrm{C} 9 \mathrm{~B}-\mathrm{C} 8 \mathrm{~B}-\mathrm{C} 13 \mathrm{~B}-\mathrm{C} 12 \mathrm{~B} & 1.0(3) \\ \mathrm{C} 11 \mathrm{C}-\mathrm{C} 12 \mathrm{C}-\mathrm{C} 13 \mathrm{C}-\mathrm{C} 14 \mathrm{C} & 178.3(2) \\ \mathrm{C} 11 \mathrm{C}-\mathrm{C} 12 \mathrm{C}-\mathrm{C} 13 \mathrm{C}-\mathrm{C} 8 \mathrm{C} & 2.0(3) \\ \mathrm{C} 7 \mathrm{C}-\mathrm{C} 8 \mathrm{C}-\mathrm{C} 13 \mathrm{C}-\mathrm{C} 14 \mathrm{C} & -0.5(3) \\ \mathrm{C} 9 \mathrm{C}-\mathrm{C} 8 \mathrm{C}-\mathrm{C} 13 \mathrm{C}-\mathrm{C} 14 \mathrm{C} & -178.42(19) \\ \mathrm{C} 7 \mathrm{C}-\mathrm{C} 8 \mathrm{C}-\mathrm{C} 13 \mathrm{C}-\mathrm{C} 12 \mathrm{C} & 176.07(19) \\ \mathrm{C} 9 \mathrm{C}-\mathrm{C} 8 \mathrm{C}-\mathrm{C} 13 \mathrm{C}-\mathrm{C} 12 \mathrm{C} & -1.9(3) \\ \mathrm{C} 12 \mathrm{~A}-\mathrm{C} 13 \mathrm{~A}-\mathrm{C} 14 \mathrm{~A}-\mathrm{C} 1 \mathrm{~A} & 176.92(18) \\ \mathrm{C} 8 \mathrm{~A}-\mathrm{C} 13 \mathrm{~A}-\mathrm{C} 14 \mathrm{~A}-\mathrm{C} 1 \mathrm{~A} & -2.1(3) \\ \mathrm{C} 12 \mathrm{~A}-\mathrm{C} 13 \mathrm{~A}-\mathrm{C} 14 \mathrm{~A}-\mathrm{C} 15 \mathrm{~A} & -6.8(3) \\ \mathrm{C} 8 \mathrm{~A}-\mathrm{C} 13 \mathrm{~A}-\mathrm{C} 14 \mathrm{~A}-\mathrm{C} 15 \mathrm{~A} & 174.26(17) \\ \mathrm{C} 2 \mathrm{~A}-\mathrm{C} 1 \mathrm{~A}-\mathrm{C} 14 \mathrm{~A}-\mathrm{C} 13 \mathrm{~A} & 177.12(18) \\ \mathrm{C} 6 \mathrm{~A}-\mathrm{C} 1 \mathrm{~A}-\mathrm{C} 14 \mathrm{~A}-\mathrm{C} 13 \mathrm{~A} & 0.0(3) \\ \mathrm{C} 2 \mathrm{~A}-\mathrm{C} 1 \mathrm{~A}-\mathrm{C} 14 \mathrm{~A}-\mathrm{C} 15 \mathrm{~A} & 0.7(3) \\ \mathrm{C} 6 \mathrm{~A}-\mathrm{C} 1 \mathrm{~A}-\mathrm{C} 14 \mathrm{~A}-\mathrm{C} 15 \mathrm{~A} & -176.46(17) \\ \mathrm{C} 12 \mathrm{~B}-\mathrm{C} 13 \mathrm{~B}-\mathrm{C} 14 \mathrm{~B}-\mathrm{C} 1 \mathrm{~B} & 175.06(18) \\ \mathrm{C} 8 \mathrm{~B}-\mathrm{C} 13 \mathrm{~B}-\mathrm{C} 14 \mathrm{~B}-\mathrm{C} 1 \mathrm{~B} & -2.7(3) \\ \mathrm{C} 12 \mathrm{~B}-\mathrm{C} 13 \mathrm{~B}-\mathrm{C} 14 \mathrm{~B}-\mathrm{C} 15 \mathrm{~B} & -8.9(3) \\ \mathrm{C} 8 \mathrm{~B}-\mathrm{C} 13 \mathrm{~B}-\mathrm{C} 14 \mathrm{~B}-\mathrm{C} 15 \mathrm{~B} & 173.37(17) \\ \mathrm{C} 2 \mathrm{~B}-\mathrm{C} 1 \mathrm{~B}-\mathrm{C} 14 \mathrm{~B}-\mathrm{C} 13 \mathrm{~B} & 178.77(18) \\ \mathrm{C} 6 \mathrm{~B}-\mathrm{C} 1 \mathrm{~B}-\mathrm{C} 14 \mathrm{~B}-\mathrm{C} 13 \mathrm{~B} & 3.4(3) \\ \end{array}$

$\begin{array}{ll}\mathrm{C} 23 \mathrm{C}-\mathrm{C} 22 \mathrm{C}-\mathrm{C} 27 \mathrm{C}-\mathrm{C} 26 \mathrm{C} & -1.7(3) \\ \mathrm{N} 2 \mathrm{C}-\mathrm{C} 22 \mathrm{C}-\mathrm{C} 27 \mathrm{C}-\mathrm{C} 26 \mathrm{C} & 176.96(19) \\ \mathrm{C} 14 \mathrm{~A}-\mathrm{C} 15 \mathrm{~A}-\mathrm{N} 1 \mathrm{~A}-\mathrm{C} 16 \mathrm{~A} & -178.47(17) \\ \mathrm{C} 21 \mathrm{~A}-\mathrm{C} 16 \mathrm{~A}-\mathrm{N} 1 \mathrm{~A}-\mathrm{C} 15 \mathrm{~A} & 141.24(19) \\ \mathrm{C} 17 \mathrm{~A}-\mathrm{C} 16 \mathrm{~A}-\mathrm{N} 1 \mathrm{~A}-\mathrm{C} 15 \mathrm{~A} & -38.8(3) \\ \mathrm{C} 14 \mathrm{~B}-\mathrm{C} 15 \mathrm{~B}-\mathrm{N} 1 \mathrm{~B}-\mathrm{C} 16 \mathrm{~B} & -176.35(17) \\ \mathrm{C} 21 \mathrm{~B}-\mathrm{C} 16 \mathrm{~B}-\mathrm{N} 1 \mathrm{~B}-\mathrm{C} 15 \mathrm{~B} & 147.60(18) \\ \mathrm{C} 17 \mathrm{~B}-\mathrm{C} 16 \mathrm{~B}-\mathrm{N} 1 \mathrm{~B}-\mathrm{C} 15 \mathrm{~B} & -31.9(3) \\ \mathrm{C} 14 \mathrm{C}-\mathrm{C} 15 \mathrm{C}-\mathrm{N} 1 \mathrm{C}-\mathrm{C} 16 \mathrm{C} & 178.31(17) \\ \mathrm{C} 17 \mathrm{C}-\mathrm{C} 16 \mathrm{C}-\mathrm{N} 1 \mathrm{C}-\mathrm{C} 15 \mathrm{C} & -137.29(19) \\ \mathrm{C} 21 \mathrm{C}-\mathrm{C} 16 \mathrm{C}-\mathrm{N} 1 \mathrm{C}-\mathrm{C} 15 \mathrm{C} & 42.3(3) \\ \mathrm{C} 18 \mathrm{~A}-\mathrm{C} 19 \mathrm{~A}-\mathrm{N} 2 \mathrm{~A}-\mathrm{C} 22 \mathrm{~A} & 165.76(19) \\ \mathrm{C} 20 \mathrm{~A}-\mathrm{C} 19 \mathrm{~A}-\mathrm{N} 2 \mathrm{~A}-\mathrm{C} 22 \mathrm{~A} & -18.0(3) \\ \mathrm{C} 27 \mathrm{~A}-\mathrm{C} 22 \mathrm{~A}-\mathrm{N} 2 \mathrm{~A}-\mathrm{C} 19 \mathrm{~A} & -12.1(3) \\ \mathrm{C} 23 \mathrm{~A}-\mathrm{C} 22 \mathrm{~A}-\mathrm{N} 2 \mathrm{~A}-\mathrm{C} 19 \mathrm{~A} & 169.9(2) \\ \mathrm{C} 20 \mathrm{~B}-\mathrm{C} 19 \mathrm{~B}-\mathrm{N} 2 \mathrm{~B}-\mathrm{C} 22 \mathrm{~B} & -16.8(3) \\ \mathrm{C} 18 \mathrm{~B}-\mathrm{C} 19 \mathrm{~B}-\mathrm{N} 2 \mathrm{~B}-\mathrm{C} 22 \mathrm{~B} & 166.81(19) \\ \mathrm{C} 27 \mathrm{~B}-\mathrm{C} 22 \mathrm{~B}-\mathrm{N} 2 \mathrm{~B}-\mathrm{C} 19 \mathrm{~B} & -11.2(3) \\ \mathrm{C} 23 \mathrm{~B}-\mathrm{C} 22 \mathrm{~B}-\mathrm{N} 2 \mathrm{~B}-\mathrm{C} 19 \mathrm{~B} & 171.4(2) \\ \mathrm{C} 18 \mathrm{C}-\mathrm{C} 19 \mathrm{C}-\mathrm{N} 2 \mathrm{C}-\mathrm{C} 22 \mathrm{C} & -6.2(3) \\ \mathrm{C} 20 \mathrm{C}-\mathrm{C} 19 \mathrm{C}-\mathrm{N} 2 \mathrm{C}-\mathrm{C} 22 \mathrm{C} & 175.27(18) \\ \mathrm{C} 23 \mathrm{C}-\mathrm{C} 22 \mathrm{C}-\mathrm{N} 2 \mathrm{C}-\mathrm{C} 19 \mathrm{C} & -12.3(3) \\ \mathrm{C} 27 \mathrm{C}-\mathrm{C} 22 \mathrm{C}-\mathrm{N} 2 \mathrm{C}-\mathrm{C} 19 \mathrm{C} & 169.19(19) \\ & \end{array}$

Hydrogen-bond geometry $\left(\AA,{ }^{\circ}\right)$

$C g 2, C g 4, C g 10, C g 11, C g 12, C g 13, C g 18, C g 20$ and $C g 21$ are the centroids of the C1A-C14A, C16A-C21A, C1B-C14B, C8B-C13B, C16B-C21B, $\mathrm{C} 22 B-\mathrm{C} 27 B, \mathrm{C} 1 C-\mathrm{C} 14 C, \mathrm{C} 22 \mathrm{C}-\mathrm{C} 27 \mathrm{C}$ and $\mathrm{C} 1 C-\mathrm{C} 8$ Crings, respectively.

\begin{tabular}{lllll}
\hline$D-\mathrm{H} \cdots A$ & $D-\mathrm{H}$ & $\mathrm{H} \cdots A$ & $D \cdots A$ & $D-\mathrm{H} \cdots A$ \\
\hline $\mathrm{C} 12 A-\mathrm{H} 9 A \cdots \mathrm{N} 1 A$ & 0.93 & 2.44 & $2.989(3)$ & 118 \\
$\mathrm{C} 12 B-\mathrm{H} 9 B \cdots \mathrm{N} 1 B$ & 0.93 & 2.47 & $3.006(3)$ & 117 \\
$\mathrm{C} 2 C-\mathrm{H} 1 C \cdots \mathrm{N} 1 C$ & 0.93 & 2.51 & $3.032(3)$ & 116 \\
$\mathrm{~N} 2 A-\mathrm{H} 20 A \cdots \mathrm{N} 1 A^{\mathrm{i}}$ & $0.92(2)$ & $2.28(2)$ & $3.147(2)$ & $158.4(18)$ \\
$\mathrm{N} 2 B-\mathrm{H} 20 B \cdots \mathrm{N} 1 C^{\mathrm{ii}}$ & $0.88(2)$ & $2.19(2)$ & $3.056(2)$ & $166.2(19)$ \\
$\mathrm{N} 2 C-\mathrm{H} 20 C \cdots \mathrm{N} 1 B$ & $0.90(2)$ & $2.22(2)$ & $3.094(2)$ & $163.2(17)$ \\
$\mathrm{C} 2 A-\mathrm{H} 1 A \cdots C g 13^{\mathrm{iii}}$ & 0.93 & 2.93 & $3.7248(3)$ & 144 \\
$\mathrm{C} 2 B-\mathrm{H} 1 B \cdots C g 21^{\text {iv }}$ & 0.93 & 2.91 & $3.6227(3)$ & 134 \\
$\mathrm{C} 4 A-\mathrm{H} 3 A \cdots C g 21^{\mathrm{v}}$ & 0.93 & 2.75 & $3.6728(3)$ & 175 \\
$\mathrm{C} 10 A-\mathrm{H} 7 A \cdots C g 12^{\mathrm{ii}}$ & 0.93 & 2.80 & $3.5075(3)$ & 134 \\
$\mathrm{C} 17 A-\mathrm{H} 11 A \cdots C g 18^{\mathrm{ii}}$ & 0.93 & 2.65 & $3.5119(3)$ & 154 \\
$\mathrm{C} 17 B-\mathrm{H} 11 B \cdots C g 10^{\mathrm{v}}$ & 0.93 & 2.79 & $3.6243(3)$ & 150 \\
$\mathrm{C} 17 B-\mathrm{H} 11 B \cdots C g 11^{\mathrm{v}}$ & 0.93 & 2.89 & $3.7248(3)$ & 150 \\
$\mathrm{C} 21 C-\mathrm{H} 13 C \cdots C g 2^{\mathrm{vi}}$ & 0.93 & 2.86 & $3.7513(3)$ & 162 \\
$\mathrm{C} 23 A-\mathrm{H} 15 A \cdots C g 2^{\mathrm{i}}$ & 0.93 & 2.85 & $3.7253(3)$ & 157 \\
$\mathrm{C} 23 B-\mathrm{H} 15 B \cdots C g 18^{\mathrm{ii}}$ & 0.93 & 2.87 & $3.7057(3)$ & 149 \\
$\mathrm{C} 25 A-\mathrm{H} 17 A \cdots C g 20^{\text {vii }}$ & 0.93 & 2.96 & $3.6262(3)$ & 130
\end{tabular}




\section{supporting information}

\begin{tabular}{lllll}
$\mathrm{C} 25 B-\mathrm{H} 17 B \cdots C g 4^{\text {viii }}$ & 0.93 & 3.00 & $3.6476(3)$ & 128 \\
$\mathrm{C} 25 C-\mathrm{H} 17 C \cdots C g 12^{\text {iv }}$ & 0.93 & 2.84 & $3.6263(3)$ & 143 \\
\hline
\end{tabular}

Symmetry codes: (i) $x,-y+3 / 2, z-1 / 2$; (ii) $x, y, z+1$; (iii) $x-1, y, z-1$; (iv) $-x+2,-y+1,-z+1$; (v) $-x+1,-y+1,-z+1$; (vi) $x, y, z-1$; (vii) $x-1,-y+3 / 2, z+1 / 2$; (viii) $x+1, y, z$. 